\title{
Relaxion and light (pseudo)scalars at the HL-LHC and lepton colliders
}

\author{
Claudia Frugiuele, Elina Fuchs, Gilad Perez and Matthias Schlaffer \\ Weizmann Institute of Science, \\ 234 Herzl Street, Rehovot 7610001, Israel \\ E-mail: Claudia.Frugiuele@weizmann.ac.il, Elina.Fuchs@weizmann.ac.il, \\ Gilad.Perez@weizmann.ac.il, Matthias.Schlaffer@weizmann.ac.il
}

ABstract: We study the potential of future lepton colliders, running at the $Z$-pole and above, and the High-Luminosity LHC to search for the relaxion and other light scalars $\phi$. We investigate the interplay of direct searches and precision observables for both $\mathcal{C} \mathcal{P}$ even and -odd couplings. In particular, precision measurements of exotic $Z$-decays, Higgs couplings, the exotic Higgs decay into a relaxion pair and associated $Z \phi$ and $\gamma \phi$ production are promising channels to yield strong bounds.

Keywords: Beyond Standard Model, CP violation, Higgs Physics

ARXIV EPRINT: 1807.10842 


\section{Contents}

1 Introduction 1

2 Relaxion phenomenology $\quad 3$

2.1 Relevant parameters 4

2.2 The relaxion-Higgs sector 5

2.3 Relaxion-gauge-boson interactions 6

$\begin{array}{lll}3 & \text { Status of experimental probes for the relaxion } & 7\end{array}$

$\begin{array}{lll}3.1 & \mathcal{C P} \text {-even couplings } & 7\end{array}$

$\begin{array}{lll}3.2 & \mathcal{C P} \text {-odd couplings } & 9\end{array}$

4 Prospects for colliders $\quad 10$

$\begin{array}{lll}4.1 & \text { Precision probes } & 11\end{array}$

4.1.1 Untagged Higgs decays 11

$\begin{array}{lll}\text { 4.1.2 Higgs self-coupling } & 14\end{array}$

$\begin{array}{lll}\text { 4.1.3 Total } Z \text { width } & 15\end{array}$

$\begin{array}{lll}4.2 & \text { Direct probes } & 16\end{array}$

$\begin{array}{lll}\text { 4.2.1 Pair production in Higgs decay } & 16\end{array}$

$\begin{array}{lll}\text { 4.2.2 } & \text { Production at the LHC } & 17\end{array}$

$\begin{array}{ll}\text { 4.2.3 Production at electron colliders } & 19\end{array}$

4.2.4 Probing the $\mathcal{C P}$-odd couplings and the relaxion CPV nature 22

4.3 Comparison of direct and indirect probes 24

5 Conclusion $\quad 26$

\section{Introduction}

The discovery of the Higgs boson in 2012 was an extraordinary success for both the Standard Model (SM) and the Large Hadron Collider (LHC). Six years later, the LHC is forcing us to reconsider our views regarding the existence of New Physics (NP) at or near the electroweak scale. Despite a rich program of dedicated searches in many channels, no conclusive signs of NP between the electroweak and the TeV scale have been found. Consequently, the idea is questioned that NP solving the hierarchy problem is at the few-TeV scale and traditional symmetry-based approaches are being challenged.

Generally speaking, constraints on new particles coupled to the SM via either the gauge interactions or other couplings of similar strength are getting increasingly strong, and push models with such couplings to non-generic corners of parameter space. As a consequence, a growing attention is now given to an alternative point of view where the 
additional degrees of freedom are light and have ultra-weak couplings to the SM, and hence are hard to find. Searching for NP of this kind can be motivated based on purely phenomenological reasonings, but it is also motivated by generic theoretical concepts that support the existence of new weakly coupled light fields. Such moduli or pseudo-NambuGoldstone-Bosons (pNGBs) appear in many extensions of the SM, see e.g. refs. [1-3] and references therein. The possibility for the presence of these new light fields is further supported by theoretical frameworks that address other conceptual and observational open questions such as the hierarchy and the strong $\mathcal{C P}$ problems or the origin of dark matter (see e.g. refs. [4-8]).

Recently, a new mechanism has been proposed that addresses the hierarchy problem in a way that goes beyond the conventional paradigm of a symmetry-based solution to fine-tuning. It belongs to the abovementioned class of models where the solution is associated with the existence of a new and special kind of pNGB, and it is worth to be examined in detail. It is denoted as the relaxion mechanism [9], where the pNGB - the relaxion - stabilizes the Higgs mass dynamically. The Higgs mass depends on the classical relaxion field value which evolves in time. The relaxion rolls down a potential, eventually stopping its rolling at a special field value where the Higgs mass is much smaller than the theory's cutoff, hence addressing the fine-tuning problem. Since the rolling needs to be very slow, a frictional force is necessary. The main sources of friction that were proposed thus far are either the Hubble friction during inflation, thereby linking the solution of the hierarchy problem to cosmology [9], or alternatively through particle production, see ref. $[10,11]$. Relaxion models do not require TeV-scale top, gauge or Higgs partners. However, they do generically lead to an interesting phenomenology. In addition to cosmological signatures $[11,12]$, relaxion models can also leave fingerprints at the low energy precision frontier, the intensity frontier and colliders $[12,13]$.

As already discussed in refs. [12, 14], the relaxion parameter space spans many orders of magnitude in mass and coupling, see figure 1. This is somewhat similar to the case of the axion, but also different in ways discussed below. The relaxion can be as light as $10^{-20} \mathrm{eV}$ (e.g. for Hubble-friction based models [9], and ignoring the relaxion's quality problem ${ }^{1}$ ) up to tens of $\mathrm{GeV}[5,12]$. This is a huge parameter space to probe and it cannot be scanned by a single experiment, or a single frontier of new physics searches. Even more so, most experiments do not even touch the physical parameter space of relaxion models. It is left to consider which of the High-Luminosity (HL)-LHC and future lepton colliders have the power to probe relaxion dynamics.

In this paper we mostly focus on searching for $\mathrm{GeV}$-scale relaxions, which are ultraheavy with respect to the relaxion framework, but at the same time at the low-mass end of the collider reach and therefore pose an experimental challenge. Admittedly, this region covers only a small part of the parameter space of the framework, however, as we shall see, future experiments will have the power to significantly probe physical regions of it. Furthermore, this region, which addresses the little hierarchy problem [15], is typically free

\footnotetext{
${ }^{1}$ In analogy to the axion quality problem, the relaxion quality problem is related to the fact that gravity does not respect global symmetries which can lead to significant modifications of the relaxion potential. For a discussion see e.g. ref. [5].
} 
of some of the model's theoretical flaws such as an exponential number of e-folds $[9,16]$, the need for significant clockworking [14, 17-19], and finally a quality problem [5, 20].

As for the present, low-mass resonances are already being searched for at the LHC, but the further development of reconstruction and analysis techniques could boost its power in this regime. Indeed, an increased effort has been invested to show that the LHC is powerful in probing several interesting BSM scenarios featuring light particles [21-27].

We focus in particular on an interesting property of the relaxion, namely that it mixes with the Higgs due to the fact that $\mathcal{C P}$ is spontaneously broken by its vacuum expectation value (vev), see e.g. refs. [5, 12]. The presence of a light new scalar mixed with the SMlike Higgs is not only a feature common to several new approaches to solve the hierarchy problem [9, 28, 29], but it is ubiquitous in many other more traditional scenarios for physics beyond the SM (BSM) such as the singlet extension [30, 31], the Higgs portal [32, 33], 2-Higgs doublet models (2HDM) [34-36], supersymmetry (SUSY) [27, 37, 38] and several others.

As a theoretically motivated framework with a light pNGB with both $\mathcal{C P}$-even and -odd couplings, the relaxion provides us with a useful benchmark model to examine the capabilities of the HL-LHC and future lepton colliders to probe the corresponding parameter space in direct and indirect channels. While our discussion mainly focuses on the relaxion framework, most of the resulting bounds also apply to the singlet extension, Higgs portal and other models.

Our paper is structured as follows. In section 2 we summarize the relaxion mechanism and highlight its couplings that are relevant for collider phenomenology. In addition, we collect in section 3 the existing bounds on the model's rather wide parameter space. In section 4 we discuss in detail direct and indirect probes of $\mathcal{C} \mathcal{P}$-even and -odd couplings, both at hadron and lepton colliders. Finally we conclude in section 5 .

\section{Relaxion phenomenology}

In the following we will briefly review the relaxion mechanism. The effective scalar potential of the theory depends both on the Higgs doublet $H$ and the relaxion $\phi$,

$$
\begin{aligned}
V(H, \phi) & =\mu^{2}(\phi) H^{\dagger} H+\lambda\left(H^{\dagger} H\right)^{2}+V_{\mathrm{sr}}(\phi)+V_{\mathrm{br}}(h, \phi), \\
\mu^{2}(\phi) & =-\Lambda^{2}+g \Lambda \phi+\ldots,
\end{aligned}
$$

where $h$ is the physical component of the Higgs doublet (before mixing with $\phi$, see below), and $\Lambda$ is the cutoff scale of a Higgs loop and. As discussed in ref. [9], $\Lambda$ needs to be significantly smaller than the Planck mass. Hence at energies above $\Lambda$, additional structure is required to fully stabilize the Higgs mass. See refs. [39-41] for attempts in UV completing relaxion models by supersymmetry and composite Higgs. During its evolution, the relaxion scans the Higgs mass parameter $\mu^{2}(\phi)$ from a large and positive cutoff energy $\Lambda^{2}$ down to negative values because of the slow-roll potential

$$
V_{\mathrm{sr}}(\phi)=r g \Lambda^{3} \phi
$$


where $g$ is a (small) dimensionless coupling which breaks the shift symmetry of the backreaction potential $V_{\mathrm{br}}$ and $r>\frac{1}{16 \pi^{2}}$ due to naturalness requirements. Once $\mu^{2}(\phi)$ becomes negative, the Higgs gets a vev $v^{2}(\phi)=-\frac{\mu^{2}(\phi)}{\lambda}$. This non-zero vev activates a backreaction potential $V_{\mathrm{br}}$ which eventually stops the rolling of the relaxion at a value $\phi_{0}$, where $v\left(\phi_{0}\right)=246 \mathrm{GeV}$.

The properties of the backreaction mechanism are model-dependent. In the minimal relaxion model discussed in ref. [9], $V_{\mathrm{br}}$ is generated by low-energy QCD and thus the relaxion is identified with the QCD axion. Yet, this setup typically predicts a too large phase $\theta_{\mathrm{QCD}}$ and is therefore ruled out by the upper bound on the neutron electric dipole moment. Possible ways to suppress the $\mathcal{C P}$ violation associated with the relaxion mechanism are discussed in refs. [9, 42]. Alternatively one can introduce a new sector with strongly [9] or weakly [15] fermions, which generates a Higgs-dependent backreaction potential of the form

$$
V_{\mathrm{br}}(h, \phi)=-\tilde{M}^{4-j}\left(\frac{v(\phi)+h}{\sqrt{2}}\right)^{j} \cos \left(\frac{\phi}{f}\right),
$$

with $1 \leq j \leq 4$ and $\tilde{M}$ being a mass parameter [12]. For later convenience we define the backreaction scale $\Lambda_{\mathrm{br}}(v(\phi))^{4} \equiv \tilde{M}^{4-j} v(\phi)^{j} / \sqrt{2}^{j}$. This scale is not predicted. It can be close to (or larger than) than the electroweak scale or as small as sub-GeV as in the QCD axion case [9], which corresponds to $j=1$. In this paper we consider only the case of $j=2$. In order to avoid fine-tuning, the backreaction scale is limited by [43]

$$
\Lambda_{\mathrm{br}}\left(v\left(\phi_{0}\right)\right)^{2} \lesssim \mathcal{O}\left(2 \pi v^{2}\right)
$$

In the following we will focus on the class of relaxion models where the backreaction has the form of eq. (2.4) and investigate its phenomenological consequences. Other realizations of the relaxion mechanism have been introduced for instance in refs. [10, 42, 43], where our analysis does not apply.

\subsection{Relevant parameters}

In refs. $[12,13]$ the couplings of the relaxion to the SM particles and their phenomenological consequences were studied. It was pointed out that generically the backreaction and/or the relaxion vev [5] lead to $\mathcal{C P}$ violation. As a result the relaxion mixes with the Higgs and inherits its couplings to SM fields.

It is convenient to write

$$
\Lambda_{\mathrm{br}}=r_{\mathrm{br}} v
$$

where $r_{\text {br }} \lesssim \sqrt{2 \pi}$ from eq. (2.5).

From the diagonalization of the $2 \times 2$ mass matrix (see ref. [12] for the full expressions) and imposing the largest eigenvalue to be the Higgs mass of $m_{h}=125 \mathrm{GeV}$ we obtain

$$
\begin{aligned}
m_{\phi}^{2} & =\frac{r_{\mathrm{br}}^{4} v^{4}\left[f^{2}\left(c_{0} m_{h}^{2}-4 r_{\mathrm{br}}^{4} v^{2} s_{0}^{2}\right)-c_{0}^{2} r_{\mathrm{br}}^{4} v^{4}\right]}{f^{2}\left(m_{h}^{2} f^{2}-c_{0} r_{\mathrm{br}}^{4} v^{4}\right)} \\
& \simeq \frac{r_{\mathrm{br}}^{4} v^{4}\left[f^{2}\left(c_{0}-16 r_{\mathrm{br}}^{4} s_{0}^{2}\right)-4 c_{0}^{2} r_{\mathrm{br}}^{4} v^{2}\right]}{f^{2}\left(f^{2}-4 c_{0} r_{\mathrm{br}}^{4} v^{2}\right)}
\end{aligned}
$$


where the approximation holds for $m_{h} \approx v / 2$. Here and in the following we denote $s_{0} \equiv$ $\sin \left(\phi_{0} / f\right), c_{0} \equiv \cos \left(\phi_{0} / f\right)$, where $\phi_{0}$ is the endpoint of the rolling of the relaxion. Moreover, to keep the notation simple, we also denote the mixed mass eigenstates by $h$ and $\phi$. In the limit of $f \gg r_{\mathrm{br}}^{2} v$, the expression for the relaxion mass is simplified to

$$
m_{\phi} \simeq \frac{r_{\mathrm{br}}^{2} v^{2}}{f} \sqrt{c_{0}-16 r_{\mathrm{br}}^{4} s_{0}^{2}}
$$

This limit requires $16 r_{\mathrm{br}}^{4} s_{0}^{2}<c_{0}$ which demands either a small backreaction scale or a suppressed $\mathcal{C P}$-violating angle $s_{0}$. This condition is easily met since for $\Lambda_{\text {br }}>v$ the endpoint of the relaxion rolling has a suppressed $s_{0}$ as clarified in ref. [13]. The mixing with the Higgs is given by

$$
\sin \theta=\left|\frac{8 f r_{\mathrm{br}}^{4} s_{0} v}{\sqrt{64 f^{2} r_{\mathrm{br}}^{8} s_{0}^{2} v^{2}+\left(f^{2}-4 c_{0} r_{\mathrm{br}}^{4} v^{2}\right)^{2}}}\right| \simeq\left|8 r_{\mathrm{br}}^{4} s_{0} \frac{v}{f}\right|,
$$

where the approximation holds in the limit of $f \gg r_{\mathrm{br}}^{2} v$. For later use we define $s_{\theta} \equiv \sin \theta$ and $c_{\theta} \equiv \cos \theta$. When $16 r_{\text {br }}^{4} s_{0}^{2} \ll c_{0}$, eq. (2.9) further simplifies to

$$
m_{\phi} \simeq \frac{r_{\mathrm{br}}^{2} v^{2}}{f} \sqrt{c_{0}} .
$$

Moreover, using eqs. (2.10) and (2.11) we find that the mixing is bounded by

$$
\sin \theta \leq 2 \frac{m_{\phi}}{v} .
$$

This bound holds within the above approximations, which are fulfilled in most of the parameter space.

\subsection{The relaxion-Higgs sector}

As discussed in ref. [12], via its mixing with the Higgs, the relaxion inherits the Higgs couplings to SM particles $g_{h X}$, where $X=f, V$ suppressed by a universal factor i.e. the mixing angle $\sin \theta$. This is precisely the case as for Higgs-portal models. The couplings of the relaxion $\phi$ to fermions $f$ and vector bosons $V$ are thus given by

$$
g_{\phi X}=\sin \theta g_{h X}
$$

The upper bound on the Higgs-relaxion mixing is of the same strength as in general Higgs portal models (see e.g. refs. $[44,45]$ ), where the mixing of $\phi$ with the Higgs as a function of the mass $m_{\phi}$ is bounded by naturalness as $\sin \theta \leq 2 \frac{m_{\phi}}{v}$, cf. eq. (2.12). In addition to the mixing, the relaxion could have a pseudoscalar coupling to fermions $\partial_{\mu} \phi \bar{f} \gamma^{\mu} \gamma^{5} f$. However, since this coupling is model dependent we omit it in the following.

Besides the couplings to SM fermions and gauge bosons, the relaxion also couples to the Higgs boson via $c_{h \phi \phi} h \phi \phi$ which is given by (see ref. [12], but here with a general $s_{0}, c_{0}$ )

$$
c_{\phi \phi h}=\frac{r_{\mathrm{br}}^{4} v^{3}}{f^{2}} c_{0} c_{\theta}^{3}-\frac{2 r_{\mathrm{br}}^{4} v^{2}}{f} s_{0} c_{\theta}^{2} s_{\theta}-\frac{r_{\mathrm{br}}^{4} v^{4}}{2 f^{3}} s_{0} c_{\theta}^{2} s_{\theta}-\frac{2 r_{\mathrm{br}}^{4} v^{3}}{f^{2}} c_{0} c_{\theta} s_{\theta}^{2}+3 v \lambda c_{\theta} s_{\theta}^{2}+\frac{r_{\mathrm{br}}^{4} v^{2}}{f} s_{0} s_{\theta}^{3} \text {. }
$$


Note that only two of the parameters $f, r_{\mathrm{br}}$ and $\theta$ are independent. In the limit of small relaxion-Higgs mixing, i.e. $\sin \theta \rightarrow 0$, the coupling $c_{\phi \phi h}$ does not vanish, but is reduced to the first term with $c_{\theta} \rightarrow 1$, originating from the backreaction potential. Furthermore, the comparison with the expression for $m_{\phi}$ in eq. (2.11) allows for $c_{0} \gg 16 r_{\mathrm{br}}^{4} s_{0}^{2}$ to express the coupling in terms of the mass as

$$
\left.c_{\phi \phi h}\right|_{\theta \rightarrow 0} \simeq \frac{r_{\mathrm{br}}^{4} v^{3}}{f^{2}} c_{0} c_{\theta}^{3} \simeq \frac{m_{\phi}^{2}}{v} .
$$

Hence the coupling becomes independent of $\theta$. This observation is reflected in the limits on $\left(m_{\phi}, \sin \theta\right)$ derived from bounds on the $c_{\phi \phi h}$ coupling from $h \rightarrow \phi \phi$ decays, see sections 4.1.1 and 4.2.1 as well as figures 2 and 5 .

Furthermore, the mixing with the relaxion modifies the Higgs self-coupling $\lambda$ with respect to its SM value of $\lambda_{\mathrm{SM}}=\frac{m_{h}^{2}}{2 v^{2}}$ (at tree level). By demanding the heavier mass eigenvalue to correctly reproduce the observed Higgs mass, $\lambda$ can be expressed as a function of $f, r_{\mathrm{br}}$ and $s_{0}$ as

$$
\lambda=\frac{-f^{2} m_{h}^{4}+c_{0} m_{h}^{2} r_{\mathrm{br}}^{4} v^{4}+4 r_{\mathrm{br}}^{8} s_{0}^{2} v^{6}}{-2 f^{2} m_{h}^{2} v^{2}+2 c_{0} r_{\mathrm{br}}^{4} v^{6}} \simeq \frac{f^{2}-4 r_{\mathrm{br}}^{4}\left(c_{0}+16 r_{\mathrm{br}}^{4} s_{0}^{2}\right) v^{2}}{8\left(f^{2}-4 c_{0} r_{\mathrm{br}}^{4} v^{2}\right)},
$$

where the simplification holds for the approximation of $m_{h} \simeq v / 2$. In addition, in order to avoid a negative $\lambda$ and other overly large contributions to Higgs-couplings, we shall take $f>1 \mathrm{TeV}$ in our numerical analysis.

\subsection{Relaxion-gauge-boson interactions}

An important ingredient for bounds on the relaxion parameter space are the couplings to the SM gauge bosons. The relaxion couples to the gauge bosons via two different classes of couplings, one that arises through its mixing with the Higgs and one that can arise due to its pNGB nature and is generically dictated by symmetry principles. The former couplings are found when $\mathcal{C P}$ violation $(\mathrm{CPV})$ is present, as discussed above, while the latter are $\mathcal{C P}$ conserving (CPC).

The leading terms in the effective Lagrangian that describes the $\mathcal{C P}$-even relaxiongauge-boson interactions are

$$
\begin{aligned}
\mathcal{L} \supset-\phi \sin \theta( & \frac{\alpha}{16 \pi v} F^{\mu \nu} F_{\mu \nu}+\frac{\alpha}{8 \pi v \tan \theta_{\mathrm{W}}} Z^{\mu \nu} F_{\mu \nu}+\frac{\alpha_{s}}{16 \pi v} G^{\mu \nu, a} G_{\mu \nu}^{a} \\
& \left.+2 \frac{m_{W}^{2}}{v} W^{+\mu} W_{\mu}^{-}+\frac{m_{Z}^{2}}{v} Z^{\mu} Z_{\mu}\right)
\end{aligned}
$$

where an order one factor that depends on $m_{\phi}$ is omitted for simplicity (see e.g. ref. [46]). If some of the SM fields couple directly to the relaxion in a manner that breaks the shift symmetry, then the above couplings will also contain non-universal pieces that cannot be described only by $\sin \theta$, a fact that leads to model dependence.

As for the pseudoscalar couplings of the relaxion to the gauge fields, these arise if the backreaction sector is anomalously charged under the corresponding gauge group, we thus 
write the effective Lagrangian as

$$
\mathcal{L} \supset \frac{\phi}{4 \pi f}\left(\frac{\tilde{c}_{\gamma \gamma}}{4} F_{\mu \nu} \widetilde{F}^{\mu \nu}+\frac{\tilde{c}_{Z \gamma}}{2} Z_{\mu \nu} \widetilde{F}^{\mu \nu}+\frac{\tilde{c}_{Z Z}}{4} Z_{\mu \nu} \widetilde{Z}^{\mu \nu}+\frac{\tilde{c}_{W W}}{4} W_{\mu \nu} \widetilde{W}^{\mu \nu}+\frac{\tilde{c}_{G G}}{4} G_{\mu \nu} \widetilde{G}^{\mu \nu}\right) .
$$

In order to compare the relevant importance of the pseudoscalar couplings to the ones induced by the mixing with the Higgs let us consider for instance the couplings to photons. For example, in cases where the coupling to photons is induced via a weakly coupled set of fermion fields, we expect $\tilde{c}_{\gamma \gamma} \sim \alpha Q_{\text {eff }}^{2}$, where $Q_{\text {eff }}$ stands for the chiral sum of the fermion charges that induces the anomaly. We note that if the backreaction sector is anomalyfree, e.g. consists of a SM gauge-neutral sector (see for example the models described in refs. $[6,15])$, then $Q_{\text {eff }}$ is suppressed by at least one additional loop factor and is expected to be rather small [12].

Two cases can be distinguished.

- There are two relevant couplings that are unique to the CPV sector: the triple scalar coupling, $c_{\phi \phi h} h \phi \phi \sim m_{\phi}^{2} / v$, given in eq. (2.15); and the relaxion coupling to $W W$ $(Z Z)$, defined in eq. (2.17) as $c_{\phi W W(Z Z)} \sim s_{\theta} 2 m_{W}^{2} / v\left(s_{\theta} 2 m_{Z}^{2} / v\right)$. These couplings generically dominate the CPC ones at colliders, in particular when one can produce at least one electroweak/Higgs boson on shell. Thus play a crucial role below.

- As for the coupling to photons, in this case both the CPV and CPC couplings are irrelevant in our effective description, and their ratio is given by

$$
\sin \theta \times \frac{f}{v} \times \frac{1}{Q_{\mathrm{eff}}^{2}} \sim \frac{r_{\mathrm{br}}^{2}}{Q_{\mathrm{eff}}^{2}}
$$

We find that the CPV coupling would dominate only for a relatively large backreaction scale, or in cases where the backreaction sector is non-anomalous.

\section{Status of experimental probes for the relaxion}

As just summarized in section 2.1, the relaxion mixes with the SM-like Higgs, leading to a scalar interaction between the relaxion $\phi$ and the SM particles. This provides the possibility to search for such a particle in various experimental setups [12, 13], depending on its mass. Its possible mass range spans several orders of magnitude from sub-eV to several tens of $\mathrm{GeV}$. Hence, it can be directly produced at high-energy colliders or be indirectly detected through its long-range, spin-independent interaction between matter constituents.

\section{$3.1 \quad \mathcal{C}$ P-even couplings}

Refs. $[12,13]$ studied the probes of the relaxion parameter space in detail. We summarize their results in the overview plot in figure 1 . The diagonal line represents the maximal mixing as allowed by eq. (2.12). One can classify the following types of probes that are relevant for the parameter space below or near this line: 
- Fifth force experiments for $m_{\phi}<10 \mathrm{eV}$. They comprise bounds from the Casimir effect $[47,48]$ (shown here) and the Eöt-Wash-type experiments $[49,50]$ for the equivalence principle [51-56] and the inverse square law [54-56] (for lower masses than shown here, see ref. [12]).

- Astrophysical bounds for $m_{\phi} \lesssim 300 \mathrm{MeV}$. They stem from red giants, horizontal branch stars [57-60] and the Supernova 1987A [61, 62].

- Beam dump experiments for $1 \mathrm{MeV}<m_{\phi} \lesssim 300 \mathrm{MeV}$. In particular proton fixed target experiments are sensitive. The presented bound is from CHARM [63-65].

- Rare meson decays for $m_{\phi} \lesssim 5 \mathrm{GeV}$. Relaxions can mediate rare decays of $K, B$ and $\Upsilon$ mesons, hence their branching ratios constrain the relaxion-Higgs mixing angle, see e.g. refs. [12, 66-68] and references therein.

- $L E P$ for $0.3 \mathrm{GeV}<m_{\phi}<116 \mathrm{GeV}$. Searches for Higgs-like particles produced in the $Z$ decay $Z \rightarrow f \bar{f} \phi$ and via Higgs-strahlung $e^{+} e^{-} \rightarrow \phi Z$ have been performed [69, 70].

- Higgs decays for $m_{\phi}<m_{h} / 2$. The LHC Run-1 set an upper limit on the untagged branching ratio of the Higgs boson [12, 71].

In addition, the relaxion is constrained by cosmological bounds on its late decays. Significant regions of the parameter space can be probed by big bang nucleosynthesis (BBN), the cosmic microwave background $(\mathrm{CMB})$ and extragalactic background light (EBL) as discussed in refs. $[12,75]$. We do not present these bounds here because they depend on various details like the reheating temperature, resulting in a strong model dependence that may even allow to circumvent them [76]. Instead, in figure 1 we show only lifetime contours to highlight where cosmological probes could play an important role, in particular $1 \mathrm{~s}$ as an indication of the beginning of $\mathrm{BBN}, 10^{17} \mathrm{~s}$ as the lifetime of the universe, and $10^{26} \mathrm{~s}$ to indicate where the relaxion is unbounded by EBL [77].

In the $10 \mathrm{eV}-1 \mathrm{MeV}$ region, only astrophysical probes are sensitive enough to probe relevant regions, i.e. below the line from eq. (2.12). Together with the bounds from invisible kaon decays shown in figure 1, neutron scattering experiments and atomic precision measurements can in principle probe Higgs portal models, but they are not yet sensitive enough to set competitive bounds [45, 78-85]. Above $1 \mathrm{MeV}$, rare meson decays, beam dump and collider experiments have sensitivity to the physical parameter region below the line.

Future experiments are being planned to improve on these existing limits. For instance both SHiP [74] and the beam dump run of NA62 [86] will extend the coverage in the MeV$\mathrm{GeV}$ mass range. Moreover, the MATHUSLA surface detector will be able to constrain the couplings of light, long-lived scalars below $m_{\phi} \simeq 5 \mathrm{GeV}$ [87, 88]. See ref. [89] for possibilities to search for long-lived particles with the ATLAS and CMS detectors. In the following we will analyse how a relaxion in the $5 \mathrm{GeV}-35 \mathrm{GeV}$ mass range can be probed by the HL-LHC and future lepton colliders. Heavier relaxions with a mass of $35 \mathrm{GeV}-62.5 \mathrm{GeV}$ are already ruled out by the present limits on untagged Higgs decays as shown in ref. [12]. Other scalars that mix with the Higgs could still exist in this mass range. 


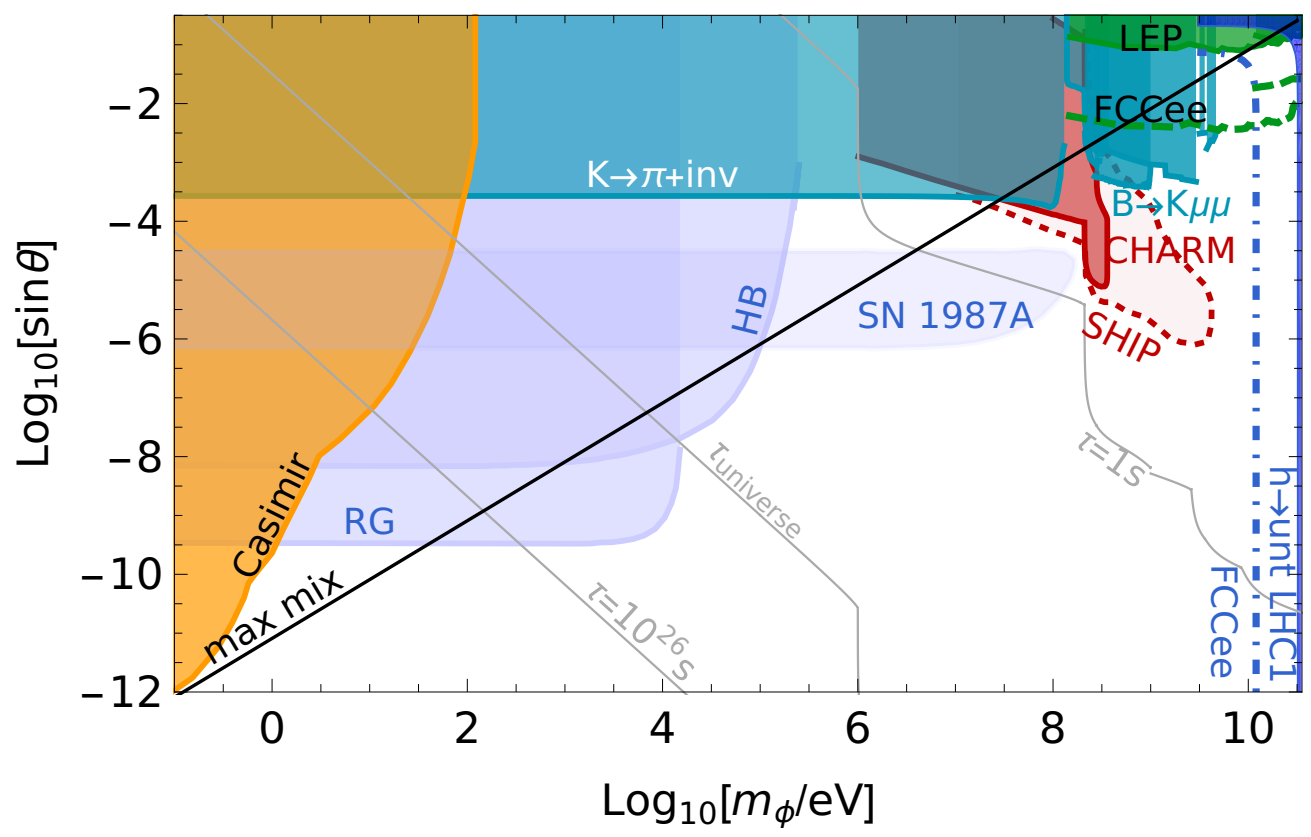

Figure 1. Summary of present bounds and few projections on the relaxion mass $m_{\phi}$ and the mixing angle $\sin \theta$ (for details see ref. [12]): fifth force via the Casimir effect (orange) [47, 48], astrophysical probes (light blue) [57-62] such as red giants (RG), horizontal branch stars (HB) and the Supernova (SN) 1987A, rare meson decays (turquoise) where the strongest bounds stem from $K \rightarrow \pi+$ invisible at E949 [66], $K_{L} \rightarrow \pi l^{+} l^{-}$at $\mathrm{KTeV} / \mathrm{E} 799[72,73]$ and $B \rightarrow K \mu^{+} \mu^{-}$at LHCb $[67,68]$. Beam dump experiment for $\phi$ production from $K$ - and $B$-decays at CHARM [63$65]$ and a projection from SHiP [74] (red dotted). Constraints from the $\phi Z$ interaction (green) via $Z \rightarrow Z^{*} \phi$ and $e^{+} e^{-} \rightarrow Z \phi$ at LEP $[69,70]$ and projections for the same processes at the FCCee (green dashed). Untagged Higgs decays (blue) at the LHC Run-1 [12] and projections for the FCCee and TeraZ (blue, dash-dotted, see section 4.1.1). The gray contours of the relaxion lifetime of $\tau_{\phi}=1 \mathrm{~s}, 10^{17} \mathrm{~s}$ and $10^{26} \mathrm{~s}$ indicate the beginning of BBN, the lifetime of the universe and safety from constraints of extragalactic background light, respectively. The black line shows the upper bound on the mixing according to eq. (2.12).

\section{$3.2 \quad \mathcal{C P}$-odd couplings}

Pseudoscalar couplings of the relaxion originate from the backreaction sector, and thus they depend on the details of the specific model as we briefly discussed in section 2.1. The perspectives to probe the relaxion via these $\mathcal{C} \mathcal{P}$-odd couplings are subject to the relative size of the $\mathcal{C} \mathcal{P}$-odd and -even couplings.

In the following we focus on the mass region above $5 \mathrm{GeV}$ and we refer to refs. [23, 9092] for a detailed discussion of the phenomenology of axion-like particles with $\sin \theta=0$. As it was shown in ref. [90], heavy-ion collisions at the LHC can provide the best limits on $\mathcal{C P}$-odd photon couplings in the $5 \mathrm{GeV}-100 \mathrm{GeV}$ mass range. Considering $\mathrm{Pb}-\mathrm{Pb}$ collisions with a luminosity of $1 \mathrm{nb}^{-1}$ yields

$$
\frac{f}{\tilde{c}_{\gamma \gamma}} \gtrsim 500 \mathrm{GeV} .
$$

LEP mono-photon searches provide a slightly weaker bound, $f / \tilde{c}_{\gamma \gamma} \gtrsim 300 \mathrm{GeV}[23]$. 
The $\phi \gamma Z$ dual coupling is instead constrained by rare $Z$ decays. For Higgs-like decays of the relaxion, the strongest limit of

$$
\frac{f}{\tilde{c}_{Z \gamma}} \gtrsim 1 \mathrm{TeV}
$$

comes from ALEPH $[93,94]$ as we derive in section 4.2.4. This decay pattern does not only appear when the Higgs-relaxion mixing dominates, but also in scenarios with $\mathcal{C} \mathcal{P}$-odd couplings to SM fermions as in ref. [5]. The case of $B R(\phi \rightarrow \gamma \gamma)=1$ was studied in ref. [91]. Similarly, the analogous $\phi Z Z$ coupling gives rise to the rare $Z$ decay $Z \rightarrow \phi Z^{*}(f \bar{f})$, and to a weak bound via the additional contribution to the total $Z$-width, $\Gamma_{Z}^{\mathrm{NP}}[91,92]$.

Furthermore, since the relaxion has both $\mathcal{C P}$-odd and -even couplings, they possibly generate a contribution to electric dipole moments (EDMs). The strongest bound comes from the electron EDM, $d_{e}$. In particular the present upper bound of $d_{e} / e \sim$ $8 \times 10^{-29} \mathrm{~cm}[95]$ implies for the product of the $\mathcal{C P}$-odd coupling to photons and $\mathcal{C P}$-even one to electrons [12],

$$
\frac{\alpha \tilde{c}_{\gamma \gamma}}{\pi} \frac{m_{e}}{v} \frac{\sin \theta}{f} \lesssim 2 \times 10^{-13} \mathrm{GeV}^{-1} \Rightarrow \frac{f}{\tilde{c}_{\gamma \gamma}}>2.5 \times 10^{4} \sin \theta \mathrm{GeV},
$$

where we omitted the logarithmic mass dependence (and, for simplicity, we omit the model dependent $\mathcal{C P}$-odd couplings to fermions here). In the coming years improvements of one order of magnitude are expected [96]. Consequently, as we will see in the following, a relaxion signal could be expected at future EDM experiments and high energy colliders, such as the HL-LHC or future electron colliders.

\section{Prospects for colliders}

After reviewing the constraints on the relaxion mass and mixing angle in the previous section, we will now identify and discuss different sensitive channels at hadron and electron colliders that are able to improve on the existing limits. The region of relaxion masses between $5 \mathrm{GeV}$ and $35 \mathrm{GeV}$ is so far only poorly constrained, but can be probed in various ways by runs with higher luminosity at the LHC as well as at future colliders. Higgs searches at LEP and constraints from untagged Higgs decays at the LHC set the so-far strongest limits in this region.

Before going to the details of the collider channels, we notice that both very low $f \ll$ $M_{\mathrm{Pl}}$ and $r_{\mathrm{br}} \lesssim \mathcal{O}(1)$ are required in order to obtain a relaxion mass in the $\mathrm{GeV}$ range [12, 13], which implies that only the little hierarchy can be addressed by the relaxion. For $m_{\phi}>$ $5 \mathrm{GeV}$, eq. (2.8) implies the following relations between the underlying model parameters:

$$
\begin{aligned}
f \geq\{1 \mathrm{TeV}, 10 \mathrm{TeV}\} & \Longrightarrow r_{\mathrm{br}} \gtrsim\{0.3,0.9\} \\
s_{0} \geq\{1 / \sqrt{2}, 0.1,0.01\} & \Longrightarrow f \lesssim\{1.5 \mathrm{TeV}, 15 \mathrm{TeV}, 150 \mathrm{TeV}\} \\
r_{\mathrm{br}} \leq\{1,1.5\} & \Longrightarrow f \lesssim\{10 \mathrm{TeV}, 30 \mathrm{TeV}\} .
\end{aligned}
$$

With $f$ and $\Lambda_{\mathrm{br}} \equiv r_{\mathrm{br}} v$ potentially within the range of future colliders, there is a chance to encounter the richer phenomenology of the UV completion of a relaxion model, beyond 
a discovery of the relaxion itself. Moreover, the heavier the relaxion, the looser the upper bound on the mixing angle $\sin \theta$, see eq. (2.12).

In the following we will use the relaxion as a benchmark model. However, our results are more universal and apply also to a general Higgs portal. The only difference is the model-dependence of the quartic Higgs coupling $\lambda$ given in eq. (2.16) and the triple-scalar coupling $c_{h \phi \phi}$ given in eq. (2.14). Hence our interpretation of the limit on the $h \rightarrow \phi \phi$ decay is relaxion-specific, while all the other presented bounds are general.

\subsection{Precision probes}

In this section we study possible constraints on the relaxion parameter space via precision measurements of Higgs and $Z$ properties, both at the HL-LHC and future lepton colliders.

\subsubsection{Untagged Higgs decays}

A powerful way to probe the relaxion at colliders is the exotic Higgs decay into a pair of relaxions, $h \rightarrow \phi \phi$ [12], exploiting the sensitivity to the triple coupling $c_{\phi \phi h}$ given in eq. (2.14). There are two complementary ways of deriving bounds from this decay. One possibility are direct searches for the decay products of the relaxions; the other one is indirect by virtue of the NP contribution to the Higgs width in a global fit of Higgs couplings. In this section we focus on the latter constraint, whereas we review the various direct searches in section 4.2.1. In figure 5 both approaches are compared, showing their strong potential in particular at lepton colliders.

Global Higgs coupling fits are sensitive to additional exotic decay channels for the Higgs that remain untagged in the corresponding searches. The measured Higgs rates allow for a global fit of the Higgs coupling modifiers $\kappa_{i}$ and the branching ratio into NP $\mathrm{BR}(h \rightarrow \mathrm{NP})$ as an additional parameter. Because the measured Higgs rates consist of the product of production and decay, the fit is model dependent. For the case of the relaxion mixed with the Higgs, a two-parameter fit is required, namely a universal modifier of the Higgs couplings to SM particles that can be identified as $\kappa \equiv \cos \theta$ (thus automatically $\left.\kappa_{V} \leq 1, V=W, Z\right)$, and $\operatorname{BR}(h \rightarrow \mathrm{NP})$ that is realized by the $h \rightarrow \phi \phi$ decay channel. The total Higgs width, neglecting loop contributions of the $\phi$, is given by

$$
\Gamma_{h}=\kappa^{2} \Gamma_{h}^{\mathrm{SM}}+\Gamma_{h}^{\mathrm{NP}} .
$$

In general, the NP contribution to the Higgs width consists of

$$
\Gamma_{h}^{\mathrm{NP}}=\Gamma_{h}^{\mathrm{inv}}+\Gamma_{h}^{\mathrm{unt}},
$$

where $\Gamma_{h}^{\text {inv }}$ denotes the partial width into invisible particles and $\Gamma_{h}^{\text {unt }}$ denotes the partial width into untagged final states that are not necessarily undetectable, but were not accounted for in the data set included in the fit, see e.g. refs. [71, 97-100]. In the relaxion case, we are interested in constraining $\Gamma_{h}^{\mathrm{NP}}=\Gamma_{h}^{\mathrm{unt}}=\Gamma(h \rightarrow \phi \phi)$, i.e. not the invisible width. With masses in the $\mathrm{GeV}$ range, the relaxion is short-lived and decays inside the detector even for small $\sin \theta$ [12]. For the case of only one universal coupling modifier $\kappa$, ref. [71] obtains $\mathrm{BR}(h \rightarrow \mathrm{NP}) \leq 20 \%$ from Run-1 data of the LHC and ref. [101] reports a 


\begin{tabular}{|l|c|r|l|c|l|l|}
\hline Collider & $\sqrt{s}[\mathrm{TeV}]$ & $\mathcal{L}_{\text {int }}\left[\mathrm{fb}^{-1}\right]$ & BR $_{\text {inv }}[\%]$ & Ref. & BR $_{\mathrm{NP}}[\%]$ & Ref. \\
\hline LHC1 & 7,8 & 22 & 37 & {$[71,101]$} & 20 & \\
LHC3 & 13 & 300 & $8.8(68 \%)$ & {$[71]$} & $7.6(68 \%)$ & {$[71]$} \\
HL-LHC & 13 & 3000 & $5.1(68 \%)$ & {$[71]$} & $4.3(68 \%)$ & \\
\hline CLIC & 0.38 & 500 & $0.97(90 \%)$ & {$[102,103]$} & 3.1 & est. \\
\hline CEPC & 0.25 & 5000 & 1.2 & {$[104]$} & 1.9 & est. \\
\hline ILC & 0.25 & 2000 & 0.3 & {$[105]$} & 1.5 & est. \\
\hline FCCee & 0.24 & 10000 & 0.19 & {$[106,107]$} & 0.64 & est. \\
\hline
\end{tabular}

Table 1. Current upper bound and projections on the branching ratios of $h \rightarrow$ invisible and $h \rightarrow \mathrm{NP}$ at various colliders running at the given center-of-mass energies $\sqrt{s}$ for benchmarks of integrated luminosity $\mathcal{L}_{\text {int }}$. Unless states otherwise in parenthesis, the bounds are given at the $95 \%$ CL. The $\mathrm{BR}(h \rightarrow \mathrm{NP})$ at future lepton colliders is estimated via eq. (4.8) and the precision on $\kappa_{Z}$ given in the text.

similar bound. This bound applies directly to the relaxion setup, as long as the modifications of the Higgs phenomenology are sufficiently described by $\sin \theta$ and the impact of the backreaction sector remains small.

Current and future runs at the (HL-)LHC as well as Higgs precision measurements at future lepton colliders will tighten the bound on untagged Higgs decays. In table 1 we collect the projections and estimates of upper bounds on $\mathrm{BR}(h \rightarrow$ untagged $)$ at the (HL-)LHC, ILC, CEPC, CLIC and FCCee running at different energies and luminosities, and compare them to the bounds on $\mathrm{BR}(h \rightarrow$ invisible $)$ from the literature.

Estimate of BR $(\boldsymbol{h} \rightarrow \mathbf{N P})$. While projections of the sensitivity to invisible Higgs decays have been published for all considered lepton colliders [71, 102-107], to our knowledge such systematic studies are still missing for Higgs decays to untagged final states. Therefore we estimate the upper bounds on $\mathrm{BR}(h \rightarrow \mathrm{NP})$ in the following way.

Due to the NP contribution to the total Higgs width in eq. (4.4), each branching ratio into SM final states $F$ is diluted as

$$
\mathrm{BR}(h \rightarrow F)=\mathrm{BR}^{\mathrm{SM}}(h \rightarrow F) \cdot[1-\mathrm{BR}(h \rightarrow \mathrm{NP})] .
$$

A bound can be set on $\operatorname{BR}(h \rightarrow \mathrm{NP})$ via the precision $\delta_{\kappa}$ of the experimental determination of $\kappa[71] .{ }^{2}$ Assuming that the observed signal rates of production times decay are SM-like within $n$ times the uncertainty, $n \cdot \delta_{\kappa}$, the modification of the production and decay must result in the product as

$$
\left(1-n \cdot \delta_{\kappa}\right)^{2} \leq \frac{\sigma_{h}}{\sigma_{h}^{\mathrm{SM}}} \cdot \frac{\mathrm{BR}(h \rightarrow F)}{\mathrm{BR}^{\mathrm{SM}}(h \rightarrow F)}=\kappa^{2} \cdot[1-\mathrm{BR}(h \rightarrow \mathrm{NP})] .
$$

Hence, the branching ratio into NP can be bounded as

$$
\mathrm{BR}(h \rightarrow \mathrm{NP}) \leq 1-\left(\frac{1-n \cdot \delta_{\kappa}}{\kappa}\right)^{2} .
$$

\footnotetext{
${ }^{2}$ We thank Tim Stefaniak for a helpful discussion regarding the approximate bounds on untagged Higgs decays at lepton colliders.
} 
Coupling fits for a model with one universal modifier have not been performed for all of the colliders considered here. Instead, several coupling modifiers for different particle species have usually been included. However, the by far most precisely determined coupling at lepton colliders is generally $\kappa_{Z}$, i.e. the coupling of $h Z Z$ due to the absolute measurement of the $Z h$ associated production cross section. Therefore this parameter dominates in the fit and is expected to yield a similar result as in a one-parameter fit. A fit of only one $\kappa$ and $\mathrm{BR}(h \rightarrow \mathrm{NP})$ would result in a stronger bound; thus the described approximation can be regarded as a conservative estimate.

In the relaxion case, $\mathrm{BR}(h \rightarrow \mathrm{NP}) \equiv \mathrm{BR}(h \rightarrow \phi \phi)$ depends on $m_{\phi}$ and $\kappa \equiv \sqrt{1-\sin ^{2} \theta}$. Thereby, as shown in figure 2 , we set constraints on $\left(m_{\phi}, \sin ^{2} \theta\right)$ approximately at the $95 \%$ CL. We obtain these by setting $n=2$ in eq. (4.8), and using the projected precision of $\kappa_{Z}$ from the various lepton colliders: $\delta_{\kappa_{Z}}^{\mathrm{CLIC}}=0.8 \%$ [102], $\delta_{\kappa_{Z}}^{\mathrm{CEPC}}=0.49 \%[104], \delta_{\kappa_{Z}}^{\mathrm{ILC}}=$ $0.38 \%[105], \delta_{\kappa_{Z}}^{\mathrm{FCCe}}=0.16 \%[106]$.

In the limit $\kappa=1$, a conservative bound on $\mathrm{BR}(h \rightarrow \mathrm{NP})$ can be derived. We summarize these bounds for future lepton colliders in table 1 (denoted by "est") derived from the precision goals of $\kappa_{Z}$. The resulting exclusion contours based on these $\kappa$-independent BR bounds are almost identical to those from constraining the product of $\kappa$ and the BR in eq. (4.7) because $\kappa$ is very close to 1 for the $\sin ^{2} \theta$ of interest and the $\kappa$-dependence of the product is dominated by the $\kappa$-dependence of $\mathrm{BR}(h \rightarrow \phi \phi)$.

For the FCCee, the Higgs BR into NP has been fitted in a multi- $\kappa$ fit without requiring $\kappa_{V} \leq 1$, resulting in $\mathrm{BR}(h \rightarrow \mathrm{NP}) \leq 0.48 \%$ at the $1 \sigma$ level [107]. The order of magnitude agrees well with our $2 \sigma$ estimate of $0.64 \%$, but due to these two restrictions we apply our result for a coherent comparability among the lepton colliders.

For the LHC, BR $(h \rightarrow \mathrm{NP})$ has been worked out in a global Higgs coupling fit for different luminosities in ref. [71]. The bound based on the Run-1 data set is explicitly calculated in the model with one universal coupling modifier and a NP branching ratio, and is also presented at the $95 \% \mathrm{CL}$. In contrast, the projections for 300 and $3000 \mathrm{fb}^{-1}$ result from a multi-dimensional $\kappa$ fit and are reported only at the $68 \%$ CL. The two modifications with respect to the Run-1 bound have opposite effects and partially compensate each other. A dedicated global fit of Higgs couplings to the single- $\kappa$ case is necessary in order to obtain 95\% CL bounds precisely applicable to the relaxion case.

Implication of the untagged Higgs decays on the relaxion parameter space. The fact that the coupling does not vanish in the limit of small $\sin \theta-$ owing to the $\cos ^{3} \theta$ term in eq. (2.15) - gives rise to a $\sin \theta$-independent bound on the mass for small $\sin ^{2} \theta \lesssim 10^{-3}$. Figure 2 shows in blue the projections arising from the $\operatorname{BR}(h \rightarrow$ untagged $)$ bounds at future colliders. While the present bound of the LHC Run 1 excludes $m_{\phi} \gtrsim 32 \mathrm{GeV}$, the HL-LHC is expected to exclude $m_{\phi} \gtrsim 20 \mathrm{GeV}$. These bounds may be significantly improved by future lepton colliders. In particular, the potential of the FCCee with $10 \mathrm{ab}^{-1}$ allows for an exclusion of $m_{\phi} \gtrsim 12 \mathrm{GeV}$. Since the $h \phi \phi$ coupling is $s_{0}$-dependent, also the exact exclusion contours depend on the endpoint of the rolling. However, by comparing numerically $s_{0}$ of $\mathcal{O}(1)$ and $\mathcal{O}\left(10^{-2}\right)$, we notice that the impact on the $\mathrm{BR}(h \rightarrow \phi \phi)$ is only very mild. Consequently, these strong bounds can be regarded as quite robust within the relaxion framework. 


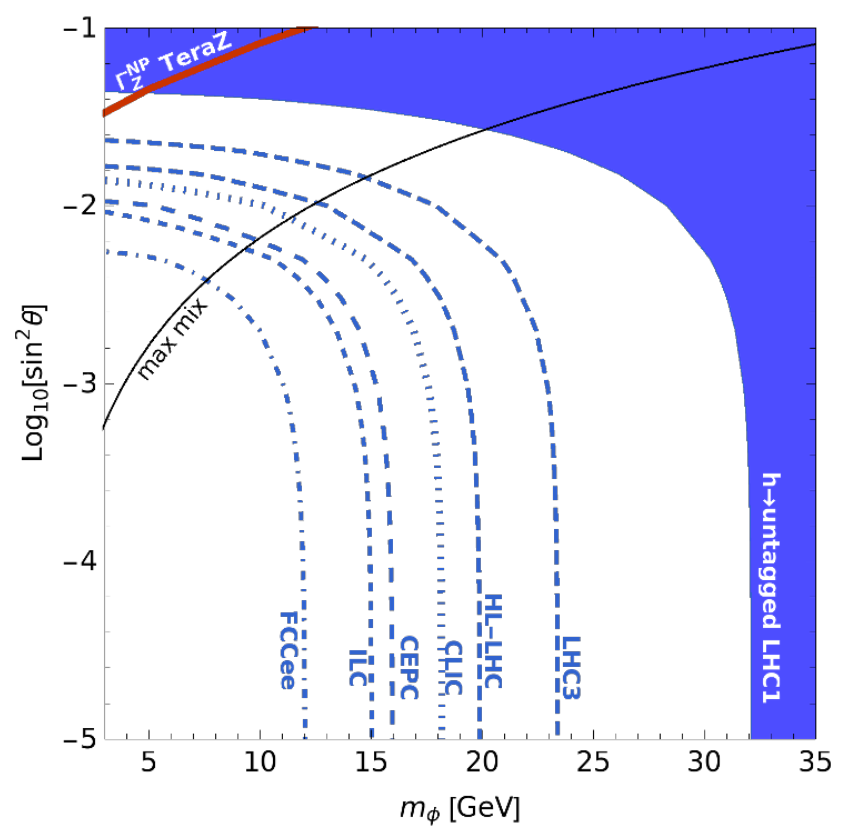

Figure 2. Precision bounds on $\sin ^{2} \theta$ and $m_{\phi}$ : upper limit on the untagged branching ratio of the Higgs boson, here $h \rightarrow \phi \phi$ (blue), obtained via the precision of Higgs couplings. Current (solid, blue area) and projected (blue, dashed) exclusion from the (HL-)LHC, CLIC at $\sqrt{s}=380 \mathrm{GeV}$ with $500 \mathrm{fb}^{-1}$, CEPC at $250 \mathrm{GeV}$ with $5 \mathrm{ab}^{-1}$, ILC with $250 \mathrm{GeV}$ with $2 \mathrm{ab}^{-1}$ and FCCee at $240 \mathrm{GeV}$ with $10 \mathrm{ab}^{-1}$. The energies, luminosities and upper bounds on $\mathrm{BR}(h \rightarrow \mathrm{NP})$ of the collider benchmarks are summarized in table 1 . The contours represent the $95 \% \mathrm{CL}$, except for LHC3 and HL-LHC which are at $68 \%$ CL. Projection of the constraint on the NP contribution $\Gamma(Z \rightarrow \phi f \bar{f})$ to the total $Z$-width, assuming the experimental precision of the FCCee running at the $Z$-pole with $10^{12} Z$ and an improved theory uncertainty (red). The black line shows the upper bound on the mixing according to eq. (2.12).

In contrast, for general Higgs portal models, the $h \phi \phi$ coupling has a different dependence on the mixing angle and accordingly, the region of $m_{\phi}>35 \mathrm{GeV}$ is not necessarily ruled out. Consequently, searches for light scalars with such masses remain highly relevant.

\subsubsection{Higgs self-coupling}

The knowledge of the the Higgs self-coupling $\lambda$ is crucial for the understanding of the Higgs potential. As shown in eq. (2.16), the Higgs-relaxion mixing alters $\lambda$. However, the sensitivity of present and future colliders to $\lambda$ via Higgs pair production is limited. While relative deviations $\Delta \lambda / \lambda=\lambda / \lambda_{\mathrm{SM}}-1$ from the SM value of $50 \%$ are estimated to be in the reach of the HL-LHC [108], the ILC might reach $10 \%$ [108] ${ }^{3}$ whereas ref. [109] obtains $-34 \%$ to $+42 \%$. According to ref. [102], CLIC is expected to become sensitive to a deviation of $\Delta \lambda / \lambda=19 \%$ for an electron polarisation of $P^{-}=-80 \%$ and combining runs at $1.4 \mathrm{TeV}$ and $3 \mathrm{TeV}$ with luminosities of $1.5 \mathrm{ab}^{-1}$ and $2 \mathrm{ab}^{-1}$, respectively, whereas ref. [109] reports $-18 \%$ to $+28 \%$ at the $68 \%$ CL. Combining the anticipated measurements at the HL-LHC and the FCCee in a 13-parameter fit, ref. [109] concludes a sensitivity of $40 \%$.

\footnotetext{
${ }^{3}$ However, this estimate of $10 \%$ at the ILC is not presented with a detailed quantitative analysis.
} 
While the estimates of the different references may not be directly comparable, it becomes clear that relatively sizeable deviations in the order of several $10 \%$ of the Higgs self-coupling from its SM prediction will remain in accordance with uncertainties at future colliders. Yet, the relaxion with a mixing of $\sin ^{2} \theta<0.1$ induces deviations from $\lambda_{\mathrm{SM}}$ of only less than $10 \%$. Consequently, the considered future colliders will not be able to constrain the relaxion parameter space via this indirect probe. A precision of $1 \%(0.1 \%)$ would be needed to constrain $\sin ^{2} \theta<10^{-2}\left(10^{-3}\right)$.

\subsubsection{Total $Z$ width}

Relaxion-mediated decays of the $Z$ boson give rise to an additional contribution $\Gamma_{Z}^{\mathrm{NP}}$ to the total $Z$ width. Since the total width measurement at LEP1 is in agreement with the theoretical SM prediction $[94,110]$, a bound on the NP contribution can be derived by limiting it to the combined experimental and theoretical uncertainty. Currently the uncertainty is dominated by the experimental one of $\delta \Gamma_{Z}^{\mathrm{LEP} 1}=2.3 \mathrm{MeV}$ [94] while the theoretical one is $\delta \Gamma_{Z}^{\text {th }}=0.5 \mathrm{MeV}$ [111]. At TeraZ, the experimental uncertainty is expected to shrink below $\delta \Gamma_{Z}^{\text {TeraZ }}=0.1 \mathrm{MeV}[107]$, necessitating further theoretical improvement. When including the so far missing 3-loop contributions, the theoretical uncertainty is estimated to reach $\delta \Gamma_{Z}^{\text {th,3-loop }}=0.2 \mathrm{MeV}$ [112]. Hence, unless the improvement of the theory uncertainty goes beyond this estimate, the theoretical and experimental uncertainties will be comparable, and we will use the combined projection of $\Gamma_{Z}^{\mathrm{NP}}<2 \times \delta \Gamma_{Z}^{\mathrm{th}+\exp } \simeq 2 \times 0.22 \mathrm{MeV}$ to set a bound at the $95 \%$ CL. Saturating this bound by the relaxion-mediated 3-body decay $Z \rightarrow \phi f \bar{f}$ for vanishing $\mathcal{C P}$-odd couplings, we obtain the constraint shown as a red line in figures 2 and 5 .

This partial width can be expressed in terms of the $\mathcal{C P}$-even and -odd couplings of the relaxion, giving rise to the integrands $\mathcal{I}, \widetilde{\mathcal{I}}$, respectively,

$$
\Gamma_{Z}^{\mathrm{NP}}=\Gamma(Z \rightarrow \phi f \bar{f})=\frac{1}{m_{Z}^{3}(2 \pi)^{3}} \sum_{f} N_{f}^{c} \int_{4 m_{f}^{2}}^{\left(m_{Z}-m_{\phi}\right)^{2}} \mathrm{~d} m_{12}^{2}(\mathcal{I}+\widetilde{\mathcal{I}}) .
$$

The sum is over the fermion final states $f$ of interest, and $m_{f}$ and $N_{f}^{c}$ are the mass and number of colors of the respective fermion. We obtain the following $\mathcal{C P}$-even and $\mathcal{C} \mathcal{P}$ odd integrands

$$
\begin{aligned}
\mathcal{I}=\frac{\mathrm{s}_{\theta}^{2}}{18} & \frac{\alpha \pi}{\cos ^{4} \theta_{\mathrm{w}} \sin ^{4} \theta_{\mathrm{w}}} \sqrt{1-\frac{4 m_{f}^{2}}{m_{12}^{2}}} \frac{\sqrt{\lambda_{\mathrm{K}}\left(m_{Z}, m_{\phi}, m_{12}\right)}}{\left(m_{12}^{2}-m_{Z}^{2}\right)^{2}+m_{Z}^{2} \Gamma_{Z}^{2}} \\
& \times\left[\left(m_{12}^{2}+2 m_{f}^{2}\right)\left(\frac{\lambda_{\mathrm{K}}\left(m_{Z}, m_{\phi}, m_{12}\right)}{m_{12}^{2}}+12 m_{Z}^{2}\right)\left(a_{f}^{2}+v_{f}^{2}\right)-72 m_{f}^{2} m_{Z}^{2} a_{f}^{2}\right], \\
\widetilde{\mathcal{I}}= & \frac{\alpha \pi}{36(4 \pi f)^{2}} \sqrt{1-\frac{4 m_{f}^{2}}{m_{12}^{2}}} \lambda_{\mathrm{K}}^{3 / 2}\left(m_{Z}, m_{\phi}, m_{12}\right)\left[\tilde{c}_{\gamma Z}^{2} Q_{f}^{2} \frac{m_{12}^{2}+2 m_{f}^{2}}{m_{12}^{4}}\right. \\
& +\frac{\tilde{c}_{Z Z}^{2}}{\sin ^{2} \theta_{\mathrm{w}} \cos ^{2} \theta_{\mathrm{w}}} \frac{\left(m_{12}^{2}+2 m_{f}^{2}\right)\left(a_{f}^{2}+v_{f}^{2}\right)-6 m_{f}^{2} a_{f}^{2}}{\left(m_{12}^{2}-m_{Z}^{2}\right)^{2}+m_{Z}^{2} \Gamma_{Z}^{2}} \\
& \left.-\frac{2 \tilde{c}_{\gamma Z} \tilde{c}_{Z Z} Q_{f} v_{f}}{\sin \theta_{\mathrm{w}} \cos \theta_{\mathrm{w}}} \frac{\left(m_{12}^{2}+2 m_{f}^{2}\right)\left(m_{Z}^{2}-m_{12}^{2}\right)}{m_{12}^{2}\left[\left(m_{12}^{2}-m_{Z}^{2}\right)^{2}+m_{Z}^{2} \Gamma_{Z}^{2}\right]}\right]
\end{aligned}
$$


respectively, with $a_{f}, v_{f}$ being the axial- and vector-coupling of the fermion, $Q$ its electric charge, $\theta_{\mathrm{W}}$ the Weinberg angle, and the Källén function is given by

$$
\lambda_{\mathrm{K}}\left(m_{1}, m_{2}, m_{3}\right)=-\left(m_{1}+m_{2}+m_{3}\right)\left(m_{1}+m_{2}-m_{3}\right)\left(m_{1}-m_{2}+m_{3}\right)\left(-m_{1}+m_{2}+m_{3}\right) .
$$

In the calculation of $\mathcal{I}$ we neglected the effect of the loop-induced $\phi Z \gamma$ interaction. We find agreement of the $\mathcal{C P}$-even contribution and the result reported in ref. [46]. For $m_{\phi}=0$ and summing over all kinematically allowed SM fermions we obtain

$$
\frac{\Gamma}{\mathrm{MeV}} \approx 23 \sin ^{2} \theta+2.1 \times 10^{-5}\left(\frac{\mathrm{TeV}}{f}\right)^{2}\left(1.1 \tilde{c}_{Z Z}^{2}-\tilde{c}_{Z Z} \tilde{c}_{\gamma Z}+47 \tilde{c}_{\gamma Z}^{2}\right)
$$

where the approximation is valid for $m_{\phi} \lesssim 0.5 \mathrm{GeV}$ for the $\mathcal{C} \mathcal{P}$-even and $m_{\phi} \lesssim 10 \mathrm{GeV}$ for the $\mathcal{C} \mathcal{P}$-odd contribution. However, the latter contribution is completely negligible in the range of interest. This justifies showing only the $\sin ^{2} \theta$ contribution in figures 2 and 5 . In principle, also other electroweak precision observables could play a role. Yet, ref. [32] showed that for the considered masses they do not constrain small enough mixing angles in the Higgs portal.

\subsection{Direct probes}

In a complementary way to the indirect bounds discussed in the previous section, the relaxion parameter space can also be constrained by direct searches in various production modes as discussed below.

\subsubsection{Pair production in Higgs decay}

While Higgs coupling fits are sensitive to the BR of $h \rightarrow \phi \phi$ irrespective of the decays of the relaxions (see section 4.1.1), one can also look directly for the relaxion decay products. Each relaxion, pair-produced in the Higgs decay, further decays into a pair of fermions $f$, photons $\gamma$ or gluons $g$ resulting in a four-particle final state $F$. ATLAS and CMS search for such signatures and report $m_{\phi}$-dependent bounds on $\frac{\sigma_{h}}{\sigma_{h}^{\mathrm{SM}}} \times \mathrm{BR}(h \rightarrow \phi \phi \rightarrow F)$, which can be compared to the prediction in the relaxion framework and thereby be translated into a bound on $\sin \theta$ and $m_{\phi}$. In table 2 we summarize the status of these exotic Higgs decay searches performed by ATLAS and CMS during Run 1 and Run 2 of the LHC, listing the final states, the considered data set and mass range of $m_{\phi}$. We conclude that none of the current searches is sensitive enough to probe parts of the relaxion parameter space displayed in figures 2 and 5, i.e. $5 \mathrm{GeV} \leq m_{\phi} \leq 35 \mathrm{GeV}$ and $10^{-5} \leq \sin ^{2} \theta \leq 10^{-1}$. Moreover, we estimate the potential reach of the HL-LHC with $3000 \mathrm{fb}^{-1}$ by rescaling the current limits by the ratio of luminosities and, in the case of Run 1 limits at $\sqrt{s}=8 \mathrm{TeV}$, additionally by the ratio of Higgs production cross sections at 8 and $13 \mathrm{TeV}$ in the dominant channels [113]. In table 2 we state the relaxion mass corresponding to the vertical asymptote of the exclusion contour in the $\left(m_{\phi}, \sin ^{2} \theta\right)$ plane.

The strongest direct bound at the HL-LHC is expected in the $b b \tau \tau$ channel excluding $m_{\phi}>26 \mathrm{GeV}$ which is presented in figure 5 (orange, dashed). The projections for $b b \mu \mu$ and $4 b$ with $V h, V=W, Z$ production are similar, but somewhat weaker (see $m_{\phi}^{\mathrm{HL}}$ in table 2 ) 
and therefore not shown in the overview plot. Neither the $\tau \tau \tau \tau$ and $\tau \tau \mu \mu$ nor the recent $\gamma \gamma g g$ final states are expected to constrain the displayed parameter plane at the HL-LHC, based on this extrapolation from the current LHC data.

The $4 \mu$ final state covers low masses of a few GeV. However, $\sin \theta$ is in part of this mass range already strongly constrained by rare $B$-decays. Furthermore, the $4 \mu$ bounds at $13 \mathrm{TeV}[114,115]$ are reported for a certain model only, namely dark gauge bosons, a 2Higgs doublet model with an additional singlet $(2 \mathrm{HDM}+\mathrm{S})$ or the NMSSM for a fixed value of $\tan \beta$, making the translation to other models less straightforward. A model-independent presentation of the updated bounds in all channels would be helpful.

As a conclusion, the only direct channels evaluated here that have the potential to constrain the displayed parameter space at the HL-LHC, are bbll, $l=\tau, \mu$ and $4 b$ in $V h$ production due to the sizeable branching fractions. However, the strongest vertical asymptote is at $m_{\phi}>26 \mathrm{GeV}$ whereas Higgs coupling fits at the HL-LHC can exclude $m_{\phi}>20 \mathrm{GeV}$. Hence at the HL-LHC, there is no direct search for $h \rightarrow \phi \phi \rightarrow F$ that is stronger than the indirect HL-LHC bound.

A different picture emerges at lepton colliders running at $\sqrt{s}=240 \mathrm{GeV}$ for which projections of the upper bound on exotic Higgs branching ratios in particular in hadronic final states have been worked out in ref. [116]. Such signatures are very hard to distinguish from background at hadron colliders, but can be promising at lepton colliders. Taking CEPC with $5 \mathrm{ab}^{-1}$ as a benchmark, a strong bound of $\mathrm{BR}(h \rightarrow \phi \phi \rightarrow 4 b)<3 \times 10^{-3}$ for $10 \mathrm{GeV} \leq m_{\phi} \leq 60 \mathrm{GeV}$ has been obtained. The resulting exclusion contour in the $\left(m_{\phi}, \sin ^{2} \theta\right)$ plane is shown in figure 5 (orange, dotted), excluding $m_{\phi}>11 \mathrm{GeV}$. Hence, this direct search is expected to constrain the relaxion parameter space more strongly than the bound derived from the anticipated precision of the Higgs couplings, see the corresponding CEPC contour in figure 2 that excludes $m_{\phi}>16 \mathrm{GeV}$. Likewise, for the FCCee and the ILC at the same energy, a similar bound on $h \rightarrow 4 b$ is expected according to their luminosities. In contrast, it is not easily transferable to CLIC due to its higher energy far above the $Z h$ threshold. A dedicated analysis would be necessary and useful to determine the reach of CLIC via these hadronic search channels of exotic Higgs decays.

\subsubsection{Production at the LHC}

Similarly to the Higgs, the dominant production modes for the relaxion at the LHC are

- gluon fusion: $p p \rightarrow \phi$

- relaxion strahlung: $p p \rightarrow Z \phi, W \phi$

- $\{t \bar{t}, b \bar{b}\}$-associated production: $p p \rightarrow\{t \bar{t} \phi, b \bar{b} \phi\}$

- vector boson fusion (VBF): $p p \rightarrow \phi j j$

In the left panel of figure 3 we present (as solid lines) the production cross sections $\sigma(p p \rightarrow$ $X$ ) depending on $m_{\phi}$ in different channels $X=\phi$ (gluon fusion), $W \phi, Z \phi, t \bar{t} \phi, b \bar{b} \phi$ and $\phi j j$ (VBF) for $\sin ^{2} \theta=1$. In all processes (beside the resonant $\phi$ production), we require a minimal transverse momentum of the relaxion of $p_{T}(\phi)>20 \mathrm{GeV}$ and evaluate the cross 


\begin{tabular}{|c|c|c|c|c|c|c|c|}
\hline$F$ & exp. & Ref. & $\sqrt{s}[\mathrm{TeV}]$ & $\mathcal{L}_{\text {int }}\left[\mathrm{fb}^{-1}\right]$ & $m_{\phi}[\mathrm{GeV}]$ & comment & $m_{\phi}^{\mathrm{HL}}[\mathrm{GeV}]$ \\
\hline$b b \tau \tau$ & CMS & {$[117]$} & 13 & 35.9 & $15-60$ & & 26 \\
\hline \multirow{2}{*}{$b b \mu \mu$} & CMS & {$[118]$} & 8 & 19.7 & $15-62.5$ & & 27 \\
& ATLAS & {$[119]$} & 13 & 36.1 & $20-60$ & & 30 \\
\hline$\tau \tau \mu \mu$ & CMS & {$[120]$} & 13 & 35.9 & $15-62.6$ & & - \\
\hline $4 \tau$ & CMS & {$[118]$} & 8 & 19.7 & $5-15$ & & - \\
\hline \multirow{2}{*}{$4 \mu$} & CMS & {$[114]$} & 13 & 2.8 & $0.25-8.5$ & NMSSM, $\gamma_{D}$ & - \\
& ATLAS & {$[115]$} & 13 & 2.8 & $1-2.5,4.5-8$ & $2 \mathrm{HDMS}, Z_{D}$ & \\
\hline \multirow{2}{*}{$4 b$} & ATLAS & {$[121]$} & 13 & 36.1 & $20-60$ & $Z h$ & 27 \\
& & & & & & $W h$ & 29 \\
\hline$\gamma \gamma g g$ & ATLAS & {$[122]$} & 13 & 36.7 & $20-60$ & VBF & - \\
\hline
\end{tabular}

Table 2. Summary of the implications of exotic Higgs decay searches on the relaxion parameter space. The columns show the final state $F$ of the search channel $p p \rightarrow h \rightarrow \phi \phi \rightarrow F$; the experiment (ATLAS, CMS) with reference; the data set collected at a center-of-mass energy $\sqrt{s}$ with an integrated luminosity of $\mathcal{L}_{\text {int }}$, the mass range of $m_{\phi}$ probed by the specific channel, comments (on the production mode and model-dependence of some bounds); estimate of the asymptotically vertical upper bound on $m_{\phi}$ of the HL-LHC projection (if any). For comparison, CEPC is expected to exclude $m_{\phi} \geq 11 \mathrm{GeV}$ via $h \rightarrow 4 b$ (for the corresponding BR, see ref. [116]).

sections at $\sqrt{s}=13 \mathrm{TeV}$. The gluon fusion cross section was calculated at $\mathrm{N}^{3} \mathrm{LO}$ and with resummation up to $\mathrm{N}^{3} \mathrm{LL}$ using ggHiggs v3.5 [123-126]. All other cross sections for processes at the HL-LHC were obtained with MadGraph5_aMC [127] at NLO. Compared to the production cross section of the Higgs with a mass of $m_{h}=125 \mathrm{GeV}$, which is of the order of pb, there is at most an enhancement of two orders of magnitude (reached only for $W \phi$ at the lowest considered $\left.m_{\phi}\right)$ for $\sin ^{2} \theta=1$ and setting all $\mathcal{C P}$-odd couplings $\tilde{c}_{i}=0$. The LEP search, however, already constrains $\sin ^{2} \theta \lesssim 10^{-2}$ in the considered mass range. As a consequence, the $\phi$ searches need to be targeted at cross sections smaller than the SM values of the $125 \mathrm{GeV}$ Higgs.

Regarding the decay modes, the right panel of figure 3 shows the branching ratios of $\phi$ for only $\mathcal{C P}$-even couplings. The leptonic channels, i.e. $\phi \rightarrow \tau \tau, \mu \mu$ have a similar branching ratio for $m_{\phi}>2 m_{b}$ as for the $125 \mathrm{GeV}$ Higgs and therefore the expected rates are small. The other clean channel, $\phi \rightarrow \gamma \gamma$, is suppressed by a factor of 100 to 2 for $m_{\phi}$ between 5 and $100 \mathrm{GeV}$, respectively, compared to the BR at a mass of $125 \mathrm{GeV}$ due to the $m_{\phi}^{3}$-dependence. Hence, an observation of this final state might only become feasible if this partial width is enhanced by the $\mathcal{C} \mathcal{P}$-odd coupling $\tilde{c}_{\gamma \gamma}$. While below the $b \bar{b}$ threshold the decay into $c \bar{c}$ is dominant, $\operatorname{BR}(\phi \rightarrow b \bar{b})$ approaches 1 where kinematically allowed. However, hadronic final states of low-mass resonances pose a severe challenge. CMS has already performed a vector resonance search with hadronic final states, yet only for $m_{\phi}>50 \mathrm{GeV}$. Further experimental efforts focused on lower masses would be helpful. Regarding the status at the LHC, CMS searched for pseudoscalars in the $25 \mathrm{GeV}-80 \mathrm{GeV}$ mass range produced in association with $b \bar{b}$ in the $19.7 \mathrm{fb}^{-1}$ data set from the $8 \mathrm{TeV}$ run (see ref. [128] for $\tau^{+} \tau^{-}$and ref. [129] for $\mu^{+} \mu^{-}$decay modes). Assuming a similar efficiency for scalars the present bound is weaker than the one from LEP. Moreover, a naive rescaling by the increased luminosity 
at HL-LHC does not improve on the LEP bound either. Nonetheless, an improvement beyond the increased luminosity might provide valuable input.

Ref. [130] investigates in a phenomenological study the discovery prospects for a new pseudoscalar with a mass between 20 and $100 \mathrm{GeV}$ produced via $t \bar{t} \phi$ with $\phi$ decaying into $b \bar{b}$. Ref. [131] instead studied the LHC phenomenology of a light scalar in the same mass range mixed with the Higgs and concluded that the LHC has the potential to slightly improve the LEP constraints for this channel for $m_{\phi}>80 \mathrm{GeV}$. However, the cross sections to be probed by this analysis are too large to be realized in a relaxion framework. Recently, the sensitivity of the LHC to light axion-like particles produced in gluon fusion has been studied for decays into $\gamma \gamma[26]$ and $\tau^{+} \tau^{-}$[22]. Their projected sensitivity could set competitive bounds for $\mathcal{C P}$-odd couplings, but for $\mathcal{C} \mathcal{P}$-even couplings the cross sections within reach correspond to a mixing angle already probed by LEP. Potentially more promising production modes are the associate production with a gauge boson, i.e. $Z \phi$ or $W \phi$, which yields larger cross sections, as shown in figure 3 .

So far we have considered the case where the dominant couplings are the ones given by the Higgs-relaxion mixing. As we previously discussed, the relaxion also has $\mathcal{C} \mathcal{P}$-odd couplings with the $\mathrm{SU}(2)$ gauge bosons which cannot only change the decay pattern, but also the production. In particular the production $p p \rightarrow W(Z) \phi$ can receive an additional contribution such as

$$
\begin{aligned}
\sigma(p p \rightarrow \phi Z)_{5} & =2.7 \times 10^{4} \mathrm{~s}_{\theta}^{2} \mathrm{fb}+2.2\left(\frac{1 \mathrm{TeV}}{f}\right)^{2}\left(1.9 \tilde{c}_{Z Z}^{2}+1.1 \tilde{c}_{Z Z} \tilde{c}_{\gamma Z}+\tilde{c}_{\gamma Z}^{2}\right) \mathrm{fb} \\
\sigma(p p \rightarrow \phi Z)_{35} & =1.0 \times 10^{4} \mathrm{~s}_{\theta}^{2} \mathrm{fb}+2.0\left(\frac{1 \mathrm{TeV}}{f}\right)^{2}\left(1.8 \tilde{c}_{Z Z}^{2}+1.0 \tilde{c}_{Z Z} \tilde{c}_{\gamma Z}+\tilde{c}_{\gamma Z}^{2}\right) \mathrm{fb} \\
\sigma(p p \rightarrow \phi W)_{5} & =6.6 \times 10^{4} \mathrm{~s}_{\theta}^{2} \mathrm{fb}+2.7\left(\frac{1 \mathrm{TeV}}{f}\right)^{2} \tilde{c}_{W W}^{2} \mathrm{fb} \\
\sigma(p p \rightarrow \phi W)_{35} & =2.3 \times 10^{4} \mathrm{~s}_{\theta}^{2} \mathrm{fb}+2.4\left(\frac{1 \mathrm{TeV}}{f}\right)^{2} \tilde{c}_{W W}^{2} \mathrm{fb},
\end{aligned}
$$

where the subscripts 5 and 35 denote $m_{\phi}$ in GeV. The partonic cross sections were calculated analytically with FeynCalc version 9.2.0 [132, 133] and folded with the LO parton distribution from NNPDF version 3.1 [134], interfaced with ManeParse version 2 [135]. As a cross check of our calculation, we compared the $\mathcal{C} \mathcal{P}$-even part with the cross section calculated with MadGraph5_aMC [127] and found agreement.

\subsubsection{Production at electron colliders}

Due to the clean environment, lepton colliders are not only able to explore the relaxion parameter space via precision measurements as discussed in the previous section, but also via direct relaxion production.

When considering the $\mathcal{C P}$-even coupling via mixing with the Higgs, there are two main production channels for relaxions at lepton colliders, $\phi Z$ and $\phi \nu_{e} \bar{\nu}_{e}$ via $W$-fusion. The cross section for the third production mode, $\phi e^{+} e^{-}$via $Z$-boson fusion, is about one order of magnitude smaller than that of $W$-fusion and will therefore only play a negligible role. 

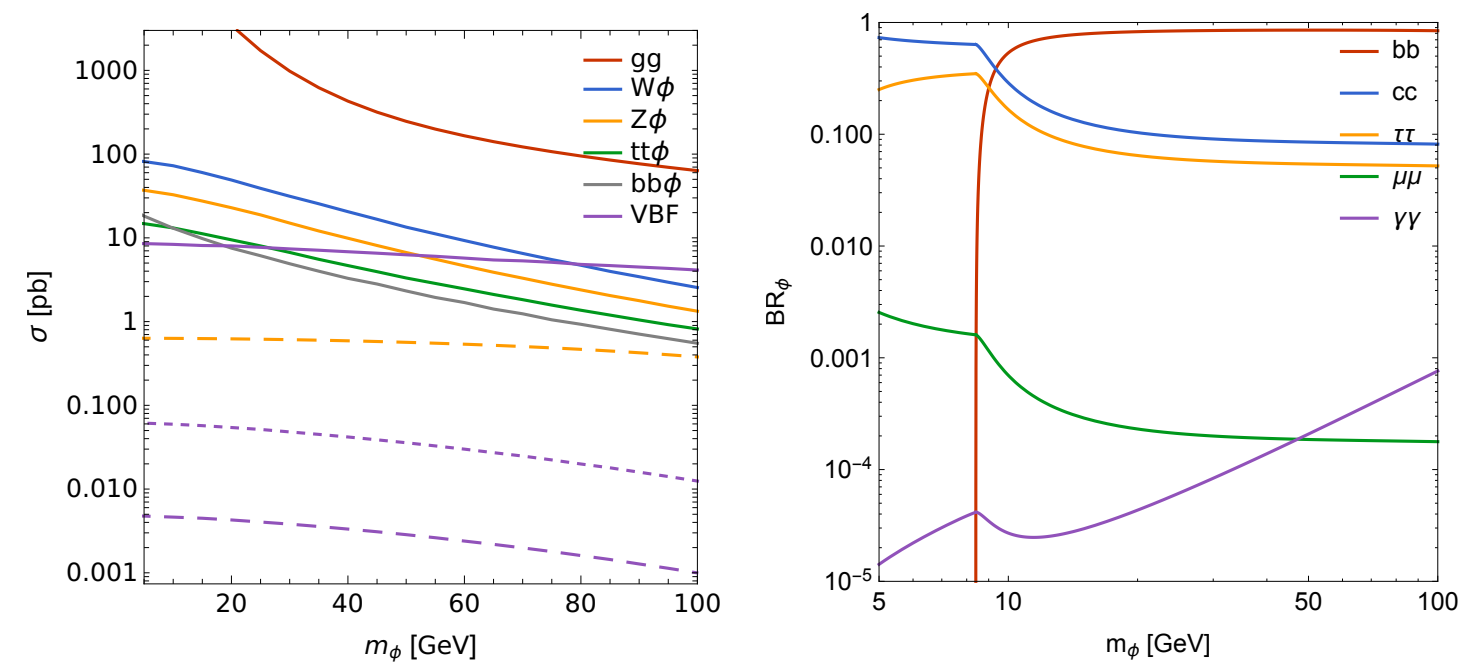

Figure 3. Production and decay of $\phi$ via $\mathcal{C P}$-even couplings for $\sin ^{2} \theta=1$. Left: hadronic cross sections in solid lines, $\sigma(p p \rightarrow X)$ at $\sqrt{s}=13 \mathrm{TeV}$ for $X=\phi$ (via gluon fusion), $W \phi, Z \phi, t \bar{t} \phi, b \bar{b} \phi$ and $\phi j j$ (via $\mathrm{VBF}$ ). Leptonic cross sections in dashed lines, $\sigma\left(e^{+} e^{-} \rightarrow Y\right.$ ) at $\sqrt{s}=240 \mathrm{GeV}$ for $Y=\phi Z, \phi \nu_{e} \bar{\nu}_{e}$ (via $W$-fusion, dotted) and $\phi e^{+} e^{-}$(via $Z$-fusion, dashed). The $\sigma(p p \rightarrow \phi)$ via gluon fusion is calculated using ggHiggs v3.5 [123-126] at $\mathrm{N}^{3} \mathrm{LO}$ including $\mathrm{N}^{3} \mathrm{LL}$ resummation without a $p_{T}$-cut. The remaining hadronic cross sections are obtained from MadGraph5_aMC [127] at NLO with $p_{T}(\phi)>20 \mathrm{GeV}$; the leptonic $\mathrm{VBF}$ cross sections at $\mathrm{LO}$ with $p_{T}\left(\phi, e^{+}, e^{-}\right)>10 \mathrm{GeV}$. The leptonic $Z \phi$ cross section was analytically calculated with eq. (4.18), also with $p_{T}(\phi)>10 \mathrm{GeV}$. Right: branching ratios $\operatorname{BR}\left(\phi \rightarrow b \bar{b}, c \bar{c}, \tau^{+} \tau^{-}, \mu^{+} \mu^{-}, \gamma \gamma\right)$.

For $\phi Z$ associated production, the polarized cross section is given by

$$
\sigma_{\phi Z}=\frac{\pi \alpha^{2} \mathrm{~s}_{\theta}^{2}}{24 s} \frac{\mathcal{P}_{P^{+} P^{-}}^{2}}{\cos ^{2} \theta_{\mathrm{w}} \sin ^{2} \theta_{\mathrm{w}}} \frac{\left[\lambda_{\mathrm{K}}\left(\sqrt{s}, m_{Z}, m_{\phi}\right)+12 m_{Z}^{2} s\right] \sqrt{\lambda_{\mathrm{K}}\left(\sqrt{s}, m_{Z}, m_{\phi}\right)}}{s\left[\left(s-m_{Z}^{2}\right)^{2}+m_{Z}^{2} \Gamma_{Z}^{2}\right]},
$$

where $P^{ \pm}$denote the positron and electron polarization, respectively, and

$$
\begin{aligned}
& \mathcal{P}_{R L}=-\frac{2 \sin \theta_{\mathrm{w}}}{\cos \theta_{\mathrm{w}}} \\
& \mathcal{P}_{L R}=\frac{1-2 \sin ^{2} \theta_{\mathrm{w}}}{\sin \theta_{\mathrm{w}} \cos \theta_{\mathrm{w}}} \\
& \mathcal{P}_{R R}=\mathcal{P}_{L L}=0,
\end{aligned}
$$

and $\lambda_{\mathrm{K}}$ is given in eq. (4.12). In the limit $s \gg m_{Z} \gg m_{\phi}$, the expression simplifies to

$$
\sigma_{\phi Z} \rightarrow \frac{\pi \alpha^{2} \mathrm{~s}_{\theta}^{2}}{24 s} \frac{\mathcal{P}_{P^{+} P^{-}}^{2}}{\cos ^{2} \theta_{\mathrm{w}} \sin ^{2} \theta_{\mathrm{w}}}\left[1+\frac{11 m_{Z}^{2}}{s}+\mathcal{O}\left(\frac{m_{\phi}^{2}}{s}, \frac{m_{Z}^{4}}{s^{2}}\right)\right] .
$$

For $\sqrt{s}=240 \mathrm{GeV}$ and $m_{\phi}=0$, the following approximate numerical values are obtained from the full expression in eq. (4.18)

$$
\sigma_{\phi Z} \approx\left\{\begin{array}{ll}
1.6 & \text { for } P^{+} P^{-}=L R \\
1.0 & \text { for } P^{+} P^{-}=R L
\end{array}\right\} \operatorname{pbs}_{\theta}^{2}
$$


The existing limits on this process from LEP2 (ALEPH, DELPHI, L3, OPAL) [70] were presented in ref. [12]. Our approach here is to estimate the reach of future lepton colliders running at the $Z$-pole or above by rescaling LEP2 bound by the square root of the ratio of luminosities. For the FCCee we assume a luminosity of $10 \mathrm{ab}^{-1}$ at a center-of-mass energy of $\sqrt{s}=240 \mathrm{GeV}$. When extrapolating the LEP2 limits, we neglect the difference in cross section due to the different center-of-mass energies within the various LEP2 runs $(192-202 \mathrm{GeV})$ and between LEP2 and the FCCee. The $\phi Z$ cross section at $\sqrt{s}=240 \mathrm{GeV}$ is by a factor of about 2 smaller than at $\sqrt{s}=\mathcal{O}(200 \mathrm{GeV})$. Yet, at the same time, also the cross sections of the background processes decrease so that our rescaling by luminosity ratios only is justified as a rough approximation.

For lighter $\phi$-masses it is more promising to consider the three-body decay $Z \rightarrow \ell \ell \phi$ at the run at the $Z$ pole. The TeraZ limit is rescaled from the LEP1 (L3) limit [69] by a factor of $\sqrt{\mathrm{N}_{Z}^{\mathrm{L} 3} / \mathrm{N}_{Z}^{\text {TeraZ }}}$ where $\mathrm{N}_{Z}^{\mathrm{L} 3}=4.4 \times 10^{6}$ and $\mathrm{N}_{Z}^{\text {TeraZ }}=10^{12}$ are the number of $Z$ bosons at the respective collider/experiment. These estimates are presented by the green dashed lines in figure 5.

This is a conservative extrapolation of cut-and-count analyses and will be certainly outperformed by modern analyses. Compared to the LEP analyses they will benefit from improved flavour-tagging techniques, better detector design and also the advances in computational methods, i.e. usage of machine-learning tools. The final states might be different from those considered in the LEP analyses when taking $\mathcal{C P}$-odd couplings to photons or to leptons into account. In order to enable an estimate of the potential of these machines independent of the relaxion decay modes, we show in figure 3 as dashed lines the unpolarized cross section $\sigma\left(e^{+} e^{-} \rightarrow Z \phi\right)$ at $\sqrt{s}=240 \mathrm{GeV}$ for $\sin ^{2} \theta=1$. The lower cross section with respect to those at the LHC will be compensated by the clean environment, the possible enhancement by polarization as well as the large planned luminosity, making future lepton colliders powerful machines for $\phi$ production.

The production of a light scalar $\phi$ with $m_{\phi}>20 \mathrm{GeV}$ in association with a $Z$ was studied in the context of the ILC in ref. [136]. In particular, for a luminosity of $\mathcal{L}=$ $2000 \mathrm{fb}^{-1}$ and with polarized beams, the LEP2 bounds can be significantly improved. Their result is shown as the green dotted line in figure 5 .

The second relevant production mode is $W$-fusion leading to a $\phi \nu_{e} \bar{\nu}_{e}$ final state. The cross section at $\sqrt{s}=240 \mathrm{GeV}$ with $p_{T}(\phi)>10 \mathrm{GeV}$ is calculated with MadGraph5_aMC yielding

$$
\begin{aligned}
\sigma\left(e^{+} e^{-} \rightarrow \phi \nu_{e} \bar{\nu}_{e}\right)_{5} & =61 s_{\theta}^{2} \mathrm{fb} \\
\sigma\left(e^{+} e^{-} \rightarrow \phi \nu_{e} \bar{\nu}_{e}\right)_{35} & =45 s_{\theta}^{2} \mathrm{fb},
\end{aligned}
$$

where the subscripts 5 and 35 denote $m_{\phi}$ in $\mathrm{GeV}$. This process played a marginal role at LEP, but it is important to be included for instance in searches targeting missing energy final states. For comparison, the cross sections for $Z$-fusion leading to the $\phi e^{+} e^{-}$final state are

$$
\begin{aligned}
\sigma\left(e^{+} e^{-} \rightarrow \phi e^{-} e^{+}\right)_{5} & =4.8 s_{\theta}^{2} \mathrm{fb} \\
\sigma\left(e^{+} e^{-} \rightarrow \phi e^{-} e^{+}\right)_{35} & =3.6 s_{\theta}^{2} \mathrm{fb}
\end{aligned}
$$


These values are calculated with an additional cut on the transverse momentum of the two leptons of $10 \mathrm{GeV}$ and the pseudorapidity $\eta<2.5$. They are about one order of magnitude smaller than the corresponding cross sections for $W$-fusion.

\subsubsection{Probing the $\mathcal{C P}$-odd couplings and the relaxion CPV nature}

We have briefly discussed above the possible (CPV) contributions of the relaxion to the electron EDM that is proportional both to its $\mathcal{C} \mathcal{P}$-even and -odd coupling. Here we consider the potential to probe the unique $\mathcal{C P}$ properties of the relaxion from processes involving its production at lepton colliders. We first discuss the case of measurements involving $\phi Z$ final states, and then move to examine those with $\phi \gamma$. As for the former channel, the production from mixing, i.e. through the $\mathcal{C} \mathcal{P}$-even vertex, is dominated by the relevant coupling to $Z$ while the latter is loop-induced and is described by a dimension-five operator in the effective field theory. This is in contrast with the pseudoscalar interactions that are always induced by dimension-five operators.

Production via $\mathcal{C P}$-odd couplings. As for the $\phi Z$ final state we have already discussed above the contribution from mixing and thus show now the production cross section via $\mathcal{C P}$-odd couplings:

$$
\begin{aligned}
\tilde{\sigma}_{\phi Z}= & \frac{\alpha}{48(4 \pi f)^{2} s} \lambda_{\mathrm{K}}\left(\sqrt{s}, m_{Z}, m_{\phi}\right)^{3 / 2} \\
& \times\left[\frac{\tilde{c}_{Z Z}^{2}}{\left(s-m_{Z}^{2}\right)^{2}+m_{Z}^{2} \Gamma_{Z}^{2}} \mathcal{P}_{P^{+} P^{-}}^{2}+\frac{4 \tilde{c}_{\gamma Z}^{2}}{s^{2}}+\frac{4 \tilde{c}_{\gamma Z} \tilde{c}_{Z Z}\left(s-m_{Z}^{2}\right)}{s\left[\left(s-m_{Z}^{2}\right)^{2}+m_{Z}^{2} \Gamma_{Z}^{2}\right]} \mathcal{P}_{P^{+} P^{-}}\right],
\end{aligned}
$$

where the polarization factors are the ones given in eq. (4.19), and for $s \gg m_{Z}^{2} \gg m_{\phi}^{2}$, it simplifies to

$$
\tilde{\sigma}_{\phi Z} \rightarrow \frac{\alpha\left(2 \tilde{c}_{\gamma Z}+\tilde{c}_{Z Z} \mathcal{P}_{P^{+} P^{-}}\right)^{2}}{48(4 \pi f)^{2}}\left[1-\frac{m_{Z}^{2}}{s} \frac{6 \tilde{c}_{\gamma Z}+\tilde{c}_{Z Z} \mathcal{P}_{P^{+} P^{-}}}{2 \tilde{c}_{\gamma Z}+\tilde{c}_{Z Z} \mathcal{P}_{P^{+} P^{-}}}+\mathcal{O}\left(\frac{m_{\phi}^{2}}{s}, \frac{m_{Z}^{4}}{s^{2}}\right)\right]
$$

For $\sqrt{s}=240 \mathrm{GeV}$ and $m_{\phi}=0$, eq. (4.26) yields

$$
\tilde{\sigma}_{\phi Z} \approx 1.0 \mathrm{fb}\left(\frac{\mathrm{TeV}}{f}\right)^{2}\left\{\begin{array}{ll}
0.60 \tilde{c}_{Z Z}^{2}+1.6 \tilde{c}_{Z Z} \tilde{c}_{\gamma Z}+\tilde{c}_{\gamma Z}^{2} & \text { for } P^{+} P^{-}=L R \\
0.39 \tilde{c}_{Z Z}^{2}-1.3 \tilde{c}_{Z Z} \tilde{c}_{\gamma Z}+\tilde{c}_{\gamma Z}^{2} & \text { for } P^{+} P^{-}=R L
\end{array} .\right.
$$

These values are about two orders of magnitude smaller than the $Z \phi$ cross section from the $\mathcal{C P}$-even interaction given in eq. (4.21), for $\tilde{c}_{i} \sim \mathrm{s}_{\theta}$. Therefore the $\mathcal{C P}$-odd interaction only plays a significant role in this process when the mixing angle is much smaller than the $\tilde{c}_{i}$ for $f=1 \mathrm{TeV}$. Allowing for both $\mathcal{C P}$-even and -odd couplings to be present, a measurement of the total cross section can only constrain one (quadratic) combination of these. However, an angular analysis of the distributions of the $Z$ decay products involving an up-down imbalance is sensitive to the $\mathcal{C} \mathcal{P}$ asymmetry of the decay (see e.g. ref. [137]). 
Consequently, one can in principle probe both the $\mathcal{C P}$-even and -odd components of the cross section.

In addition to the $Z \phi$ production we also consider the $\gamma \phi$ production. We first note that for the latter the mixing contributions only arise at the one-loop level and thus this channel is much less sensitive to these when compared to the $Z \phi$ channel. For simplicity we therefore focus on the $\mathcal{C P}$-odd couplings with the corresponding cross section given by

$$
\tilde{\sigma}_{\phi \gamma}=\frac{\alpha\left(s-m_{\phi}^{2}\right)^{3}}{48 s(4 \pi f)^{2}}\left[\frac{\tilde{c}_{\gamma Z}^{2} \mathcal{P}_{P^{+} P^{-}}^{2}}{\left(s-m_{Z}^{2}\right)^{2}+m_{Z}^{2} \Gamma_{Z}^{2}}+\frac{4 \tilde{c}_{\gamma \gamma}^{2}}{s^{2}}+\frac{4 \tilde{c}_{\gamma Z} \tilde{c}_{\gamma \gamma}\left(s-m_{Z}^{2}\right) \mathcal{P}_{P^{+} P^{-}}}{s\left[\left(s-m_{Z}^{2}\right)^{2}+m_{Z}^{2} \Gamma_{Z}^{2}\right]}\right]
$$

where the polarization factors $\mathcal{P}_{P^{+} P^{-}}$are given in eq. (4.19). In the limit $s \gg m_{Z}^{2} \gg m_{\phi}^{2}$ it simplifies to

$$
\tilde{\sigma}_{\phi \gamma} \rightarrow \frac{\alpha\left(2 \tilde{c}_{\gamma \gamma}+\tilde{c}_{\gamma Z} \mathcal{P}_{P^{+} P^{-}}\right)^{2}}{48(4 \pi f)^{2}}\left[1+\frac{m_{Z}^{2}}{s} \frac{2 \tilde{c}_{\gamma Z} \mathcal{P}_{P^{+} P^{-}}}{2 \tilde{c}_{\gamma \gamma}+\tilde{c}_{\gamma Z} \mathcal{P}_{P^{+} P^{-}}}+\mathcal{O}\left(\frac{m_{\phi}^{2}}{s}, \frac{m_{Z}^{4}}{s^{2}}\right)\right]
$$

which agrees with the result presented in ref. [91] within their assumptions. For $\sqrt{s}=$ $240 \mathrm{GeV}$ and $m_{\phi}=0$, eq. (4.29) yields

$$
\tilde{\sigma}_{\phi \gamma} \approx 1.6 \mathrm{fb}\left(\frac{\mathrm{TeV}}{f}\right)^{2}\left\{\begin{array}{ll}
0.6 \tilde{c}_{\gamma Z}^{2}+1.6 \tilde{c}_{\gamma \gamma} \tilde{c}_{\gamma Z}+\tilde{c}_{\gamma \gamma}^{2} & \text { for } P^{+} P^{-}=L R \\
0.39 \tilde{c}_{\gamma Z}^{2}-1.3 \tilde{c}_{\gamma \gamma} \tilde{c}_{\gamma Z}+\tilde{c}_{\gamma \gamma}^{2} & \text { for } P^{+} P^{-}=R L
\end{array} .\right.
$$

Finally, rare $Z$ decays into $\phi \gamma$ involve $\tilde{c}_{\gamma Z}$. For this partial width we obtain

$$
\widetilde{\Gamma}(Z \rightarrow \phi \gamma)=\tilde{c}_{\gamma Z}^{2} \frac{m_{Z}^{3}}{96 \pi(4 \pi f)^{2}}\left(1-\frac{m_{\phi}^{2}}{m_{Z}^{2}}\right)^{3}
$$

in agreement with e.g. ref. [91].

Constraining the $\mathcal{C P}$-odd couplings. The above cross section prediction for $\tilde{\sigma}_{\phi Z}$ as well as the decay widths of $Z \rightarrow \ell \ell \phi$ and $Z \rightarrow \phi \gamma$ can be used to constrain the $\mathcal{C P}$-odd couplings $\tilde{c}_{Z Z}$ and $\tilde{c}_{\gamma Z}$. We evaluate the implications of the LEP measurements at and above the $Z$-pole and estimate the sensitivity at TeraZ and the FCCee.

Regarding $Z \rightarrow \ell \ell \phi$, we translate the L3 bound from LEP1 [69] on $\sin ^{2} \theta$ shown in figure 5 into bounds on the $\mathcal{C P}$-odd $\tilde{c}_{Z Z}$ coupling. For this we require

$$
\mathrm{BR}_{Z \rightarrow \ell \ell \phi}\left(\sin ^{2} \theta_{\max }\right) \stackrel{!}{=} \widetilde{\mathrm{BR}}_{Z \rightarrow \ell \ell \phi}\left(\left(\tilde{c}_{Z Z} / f\right)_{\max }^{2}\right)
$$

i.e. ignoring differences in the distributions. Here BR refers to the decay through mixing where all $\mathcal{C} \mathcal{P}$-odd couplings are neglected whereas $\widetilde{\mathrm{BR}}$ refers to the decay based on the $\mathcal{C P}$-odd coupling $\tilde{c}_{Z Z}$. For TeraZ, we take the rescaled LEP1 limit on $\sin ^{2} \theta$.

Likewise we proceed for $Z \rightarrow \phi \gamma$ where only $\tilde{c}_{\gamma Z}$ plays a role. The strongest bound on $\mathrm{BR}(Z \rightarrow \phi \gamma) \times \mathrm{BR}(\phi \rightarrow f f)$ is reported by ALEPH [93] for $f=\tau$. Here we assume the decay of $Z$ into $\phi \gamma$ via $\tilde{c}_{\gamma Z}$ and the decay of the relaxion into $\tau \tau$ via mixing with the BR as shown in figure 3 . Since $\operatorname{BR}(\phi \rightarrow f f)$ is independent of $\sin ^{2} \theta$, this approach is valid for 

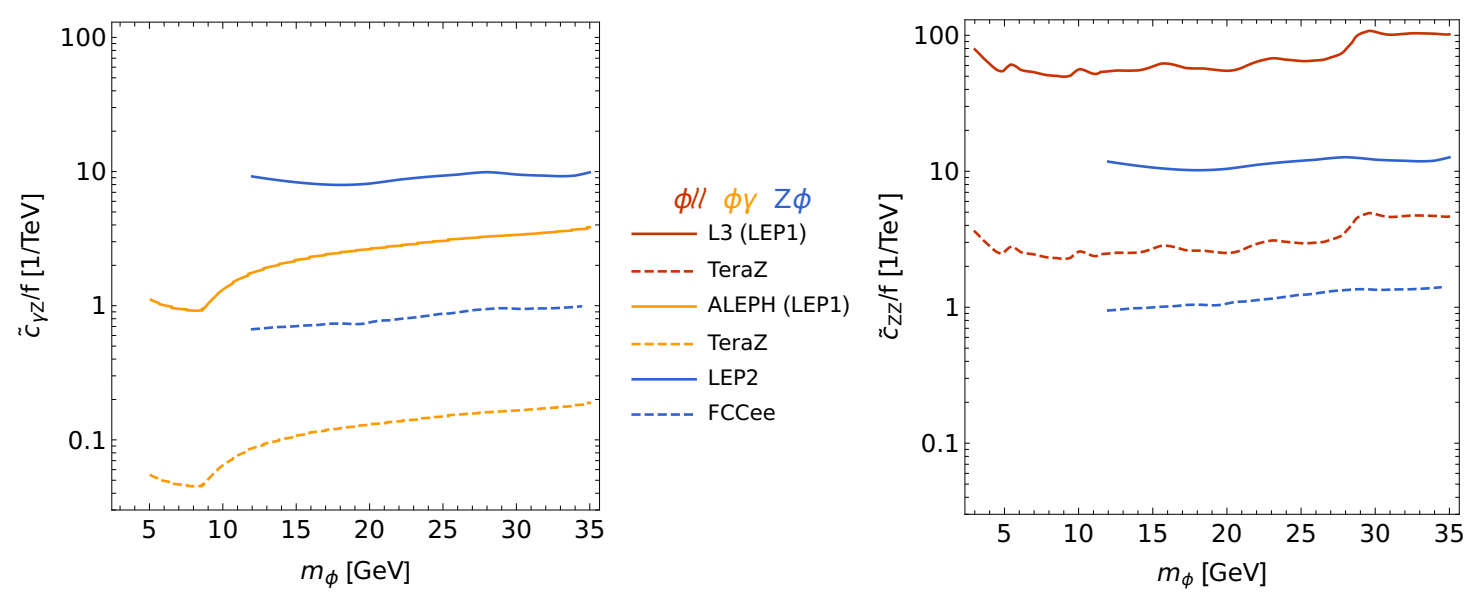

Figure 4. Existing (solid) and projected (dashed) limits on the $\mathcal{C P}$-odd coupling of the relaxion to $\gamma Z$ (left) and $Z Z$ (right) as a function of the relaxion mass: $e^{+} e^{-} \rightarrow Z \phi$ (blue) at LEP2 [70] and FCCee. Additionally for $\tilde{c}_{\gamma Z} / f: Z \rightarrow \gamma \phi$ (orange) at ALEPH [93, 94] during LEP1 and at TeraZ. Moreover for $\tilde{c}_{Z Z} / f: \Gamma(Z \rightarrow \ell \ell \phi)$ (red) at L3 during LEP1 [69] and TeraZ.

sufficiently small $\sin ^{2} \theta$. We rescale the ALEPH limit by the ratio of $Z$ bosons used in the analysis [94] to the $10^{12}$ expected at TeraZ.

Concerning $e^{+} e^{-} \rightarrow \phi Z$, we analogously require the number of $Z$ bosons, $N_{Z}$, produced in this channel via $\tilde{c}_{i}$ to be equal to $N_{Z}$ produced via mixing at the $\sin ^{2} \theta$ that is maximally allowed by LEP2 or its rescaling to FCCee, respectively. In order to obtain $N_{Z}$ at LEP2, we sum over the products of luminosity times cross section evaluated at the energies of the respective runs [138].

Figure 4 shows the current limits and future projections on $\tilde{c}_{\gamma Z}$ and $\tilde{c}_{Z Z}$. While the runs around $200 \mathrm{GeV}$ set stronger bounds on $\tilde{c}_{Z Z}$ than those at $m_{Z}, \tilde{c}_{\gamma Z}$ is best constrained at the $Z$-pole.

Finally at very high energy lepton colliders like the high-energy stage of CLIC, $W$ boson fusion becomes an important process to produce axion-like particles [139]. This could be used to set bounds on $\tilde{c}_{W W}$.

\subsection{Comparison of direct and indirect probes}

The comparison of the direct and indirect probes in the summary plot of figure 5 highlights the complementarity and perspectives in exploring the relaxion framework at colliders. There are ample regions of parameter space where the relaxion can be discovered both via deviations in precision measurements of Higgs properties and via direct production.

Focusing on the discovery potential of the future colliders, we notice that the searches for Higgs decays into a pair of relaxions can probe relaxion masses rather independently of the mixing angle. Such bounds can be complemented by direct searches in other channels and confirm or rule out a potential indirect evidence. Moreover, direct searches are the only way to explore relaxion masses below $20 \mathrm{GeV}$ at the (HL-)LHC, providing a strong motivation to try to push the sensitivity below this mass. In this context, $\tau$ final states are particularly motivated due to the large branching ratio in Higgs portal models and 


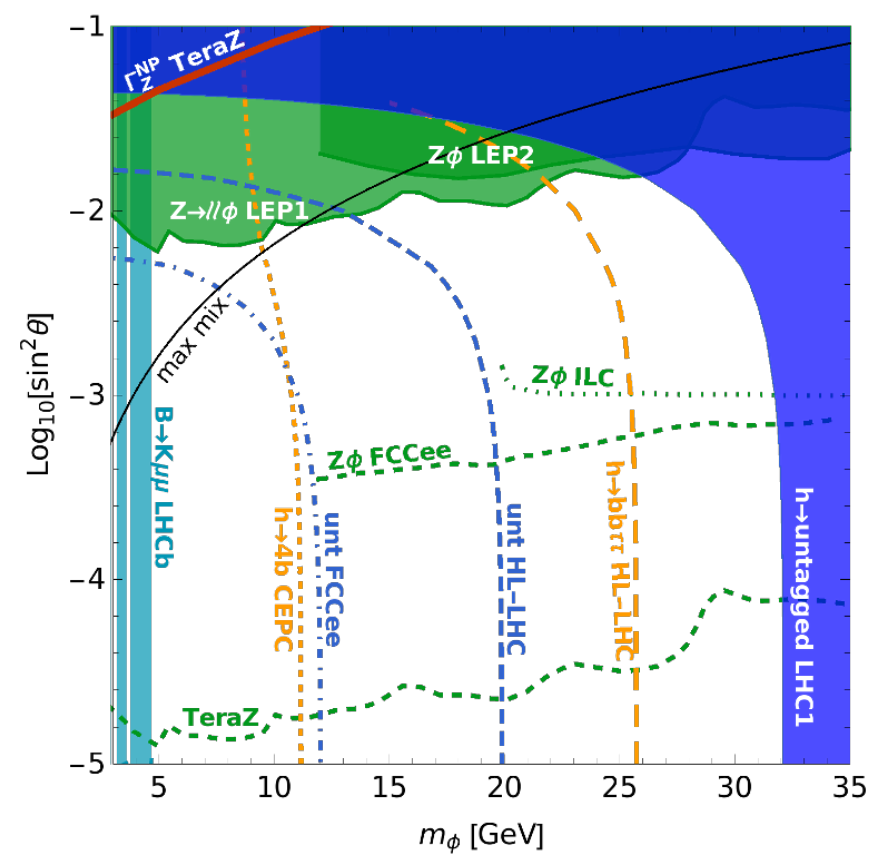

Figure 5. Direct and indirect bounds and projections for processes at hadron and lepton colliders. $Z \rightarrow Z^{*} \phi \rightarrow \ell \bar{\ell} \phi$ at LEP1 with $\sqrt{s}=M_{Z}$ [69] and $e^{+} e^{-} \rightarrow Z \phi$ at LEP2 with $\sqrt{s}=192$ $202 \mathrm{GeV}$ [70]; projections for the same processes at the FCCee (green, dashed) running at $\sqrt{s}=M_{Z}$ with $10^{12} Z$ s produced (TeraZ) and $\sqrt{s}=240 \mathrm{GeV}$ with $10 \mathrm{ab}^{-1}$. Projection for $e^{+} e^{-} \rightarrow Z \phi$ at the ILC with $\mathcal{L}_{\text {int }}=2 \mathrm{ab}^{-1}$ [136] (green, dotted). Bound from $B^{+} \rightarrow K^{+} \mu^{+} \mu^{-}$at LHCb [67, 68]. Direct searches for exotic Higgs decays at the HL-LHC in the $b b \tau \tau$ channel inferred from ref. [117] (orange, dashed) and at CEPC with $5 \mathrm{ab}^{-1}$ in the $4 b$ channel from the BR bound of ref. [116]. Untagged Higgs decays (blue) at the LHC Run-1 (blue area) and projections for the HL-LHC with $3 \mathrm{ab}^{-1}$ (blue, dashed) and the FCCee with $10 \mathrm{ab}^{-1}$ (blue, dash-dotted) according to table 1 . The NP contribution to the total $Z$-width will be bounded by TeraZ (red). The maximal mixing according to eq. (2.12) is indicated by the black line.

a reasonable reconstruction efficiency. At the price of a lower branching ratio, but with the benefit of a cleaner signature, also other leptonic final states should be explored, see figure 3.

Despite the precision at TeraZ and the expected improvement of the theoretical uncertainty, the NP contribution to the total $Z$-width will not set new limits beyond the already excluded range. Nevertheless, direct searches at TeraZ for the decay of $Z \rightarrow \phi f \bar{f}$ do play an important role in constraining a light $\phi$.

The 'collider region' of the relaxion is bounded from below by the LHCb bound of up to $5 \mathrm{GeV}$. While for general scalars any higher masses are relevant, the relaxion window ends at $32 \mathrm{GeV}$ on account of the bound set by untagged Higgs decays.

The maximal relaxion-Higgs mixing angle as a function of the mass is limited by eq. (2.12) indicated by the black line. Hence the current bounds hardly probe the viable region whereas the projections for all of the considered future colliders cut deeply into this physical parameter region. 


\section{Conclusion}

In this paper we evaluated the potential sensitivity of future colliders to light scalars, motivated by the relaxion framework. The relaxion phenomenology is driven by two kinds of couplings: the first kind is in their simplest form identical to those of a $\mathcal{C P}$-even Higgs portal and originates from the relaxion mixing with the Higgs; the second kind is similar to those of $\mathcal{C P}$-odd axion-like-particles and typically arises due to an anomalous backreaction sector. We studied indirect effects and direct production modes both at the HL-LHC and at future lepton colliders, namely the ILC, CLIC, the CEPC and the FCCee running at the $Z$-pole and above the $Z h$ production threshold. Our results are applicable to a large class of models beyond the relaxion framework. Light (pseudo-)scalars can arise in a variety of models, for example Higgs portals, 2HDM with an additional singlet, and supersymmetric versions thereof, as well as in models with axion-like particles.

The fact that the relaxion acquires both $\mathcal{C P}$-even and -odd couplings makes it an interesting model to study, in particular given the powerful capabilities of future colliders. While focusing mainly on the implications of the $\mathcal{C P}$-even interactions, we also point out in which channels the $\mathcal{C P}$-odd couplings can influence the collider phenomenology and derive constraints on them.

Among the indirect probes, we evaluated the NP contribution to the total $Z$-width, in this case from the decay $Z \rightarrow f \bar{f} \phi$, whose current bound from LEP1 will be significantly improved at TeraZ. Furthermore, we studied the sensitivity to the $h \phi \phi$ coupling via untagged $h \rightarrow \phi \phi$ decays, resulting in strong, though relaxion-specific bounds.

Regarding the direct probes, we considered gluon fusion, associated production as well as explicit searches for $h \rightarrow \phi \phi$. We provided analytic expressions for polarized cross sections at lepton colliders and semi-analytic functions for the processes at the HL-LHC, involving both the $\mathcal{C P}$-even and -odd couplings.

Our main findings are:

- We chart the physical parameter region of relaxion models, expressed via the relaxionHiggs mixing angle as a function of the mass. We find that only mixing angles smaller than twice the ratio of mass to the Higgs vacuum expectation value can describe actual relaxion models. It is quite interesting that the HL-LHC and future colliders are capable to significantly probe this "physical relaxion" region, albeit only for a very massive relaxion.

- The HL-LHC can probe relaxion masses roughly above $20 \mathrm{GeV}$ by untagged Higgs decays. However, its discovery prospects in direct channels via its mixing with the Higgs are limited due to the large background for low-mass resonances and suppressed branching ratios for the clean final states.

- Future lepton colliders have the potential to significantly improve on existing limits and HL-LHC projections on the parameter space via direct and indirect channels. In particular masses roughly above $10 \mathrm{GeV}$, and mixing angles above few times $10^{-3}$ will be probed by exotic Higgs and exclusive $Z$ decays respectively. 
- The $\mathcal{C P}$-nature of $\phi$ can be deciphered by the interplay of $\mathcal{C P}$-even and -odd driven signals. In particular the interplay of collider observables (such as $\phi Z$ and possibly $\phi \gamma$ production as well as the angular distribution of decay products) and EDMs is crucial.

\section{Acknowledgments}

We would like to thank Liron Barak, Roberto Franceschini, Rick S. Gupta, Marumi Kado, Yevgeny Kats, Yosef Nir, Diego Redigolo, Francesco Spano and Tim Stefaniak for useful discussions. The work of GP is supported by grants from the BSF, ERC, ISF, Minerva Foundation, and the Weizmann-U.K. Making Connections Program. EF is supported by the Minerva Foundation.

Open Access. This article is distributed under the terms of the Creative Commons Attribution License (CC-BY 4.0), which permits any use, distribution and reproduction in any medium, provided the original author(s) and source are credited.

\section{References}

[1] A. Arvanitaki et al., String axiverse, Phys. Rev. D 81 (2010) 123530 [arXiv:0905.4720] [INSPIRE].

[2] R. Essig et al., Working group report: new light weakly coupled particles, in the proceedings of the 2013 Community Summer Study on the Future of U.S. Particle Physics: Snowmass on the Mississippi (CSS2013), July 29-August 6, Minneapolis, U.S.A. (2013), arXiv: 1311.0029 [INSPIRE].

[3] J. Alexander et al., Dark sectors 2016 workshop: community report, arXiv:1608.08632 [INSPIRE].

[4] R.D. Peccei and H.R. Quinn, CP conservation in the presence of instantons, Phys. Rev. Lett. 38 (1977) 1440 [INSPIRE].

[5] O. Davidi et al., The Nelson-Barr relaxion, arXiv:1711.00858 [INSPIRE].

[6] O. Davidi et al., The hierarchion, a relaxion addressing the standard model's hierarchies, JHEP 08 (2018) 153 [arXiv:1806.08791] [INSPIRE].

[7] W. Hu, R. Barkana and A. Gruzinov, Cold and fuzzy dark matter, Phys. Rev. Lett. 85 (2000) 1158 [astro-ph/0003365] [INSPIRE].

[8] F. Wilczek, Axions and family symmetry breaking, Phys. Rev. Lett. 49 (1982) 1549 [INSPIRE].

[9] P.W. Graham, D.E. Kaplan and S. Rajendran, Cosmological relaxation of the electroweak scale, Phys. Rev. Lett. 115 (2015) 221801 [arXiv: 1504.07551] [INSPIRE].

[10] A. Hook and G. Marques-Tavares, Relaxation from particle production, JHEP 12 (2016) 101 [arXiv: 1607.01786] [INSPIRE].

[11] N. Fonseca, E. Morgante and G. Servant, Higgs relaxation after inflation, JHEP 10 (2018) 020 [arXiv: 1805.04543 ] [INSPIRE].

[12] T. Flacke et al., Phenomenology of relaxion-Higgs mixing, JHEP 06 (2017) 050 [arXiv: 1610.02025] [INSPIRE]. 
[13] K. Choi and S.H. Im, Constraints on relaxion windows, JHEP 12 (2016) 093 [arXiv: 1610.00680] [INSPIRE].

[14] K. Choi and S.H. Im, Realizing the relaxion from multiple axions and its UV completion with high scale supersymmetry, JHEP 01 (2016) 149 [arXiv:1511.00132] [INSPIRE].

[15] R.S. Gupta, Z. Komargodski, G. Perez and L. Ubaldi, Is the relaxion an axion?, JHEP 02 (2016) 166 [arXiv: 1509.00047] [inSPIRE].

[16] S.P. Patil and P. Schwaller, Relaxing the electroweak scale: the role of broken dS symmetry, JHEP 02 (2016) 077 [arXiv: 1507.08649] [INSPIRE].

[17] K. Choi, H. Kim and S. Yun, Natural inflation with multiple sub-Planckian axions, Phys. Rev. D 90 (2014) 023545 [arXiv:1404.6209] [InSPIRE].

[18] D.E. Kaplan and R. Rattazzi, Large field excursions and approximate discrete symmetries from a clockwork axion, Phys. Rev. D 93 (2016) 085007 [arXiv:1511.01827] [InSPIRE].

[19] G.F. Giudice and M. McCullough, A clockwork theory, JHEP 02 (2017) 036 [arXiv:1610.07962] [INSPIRE].

[20] R.S. Gupta, Relaxion measure problem, Phys. Rev. D 98 (2018) 055023 [arXiv: 1805.09316] [INSPIRE].

[21] CMS collaboration, Search for low mass vector resonances decaying into quark-antiquark pairs in proton-proton collisions at $\sqrt{s}=13 \mathrm{TeV}, \mathrm{JHEP} 01$ (2018) 097 [arXiv:1710.00159] [INSPIRE].

[22] G. Cacciapaglia, G. Ferretti, T. Flacke and H. Serodio, Revealing timid pseudo-scalars with taus at the LHC, Eur. Phys. J. C 78 (2018) 724 [arXiv:1710.11142] [InSPIRE].

[23] J. Jaeckel and M. Spannowsky, Probing MeV to $90 \mathrm{GeV}$ axion-like particles with LEP and LHC, Phys. Lett. B 753 (2016) 482 [arXiv:1509.00476] [InSPIRE].

[24] D. Curtin, R. Essig, S. Gori and J. Shelton, Illuminating dark photons with high-energy colliders, JHEP 02 (2015) 157 [arXiv:1412.0018] [INSPIRE].

[25] U. Haisch, J.F. Kamenik, A. Malinauskas and M. Spira, Collider constraints on light pseudoscalars, JHEP 03 (2018) 178 [arXiv:1802.02156] [INSPIRE].

[26] A. Mariotti, D. Redigolo, F. Sala and K. Tobioka, New LHC bound on low-mass diphoton resonances, Phys. Lett. B 783 (2018) 13 [arXiv:1710.01743] [INSPIRE].

[27] B. Bellazzini et al., R-axion at colliders, Phys. Rev. Lett. 119 (2017) 141804 [arXiv:1702.02152] [INSPIRE].

[28] N. Arkani-Hamed et al., Solving the hierarchy problem at reheating with a large number of degrees of freedom, Phys. Rev. Lett. 117 (2016) 251801 [arXiv:1607.06821] [INSPIRE].

[29] A. Hook, Solving the hierarchy problem discretely, Phys. Rev. Lett. 120 (2018) 261802 [arXiv: 1802.10093] [INSPIRE].

[30] T. Robens and T. Stefaniak, LHC benchmark scenarios for the real Higgs singlet extension of the standard model, Eur. Phys. J. C 76 (2016) 268 [arXiv:1601.07880] [InSPIRE].

[31] I.M. Lewis and M. Sullivan, Benchmarks for double Higgs production in the singlet extended standard model at the LHC, Phys. Rev. D 96 (2017) 035037 [arXiv: 1701.08774] [INSPIRE].

[32] A. Falkowski, C. Gross and O. Lebedev, A second Higgs from the Higgs portal, JHEP 05 (2015) 057 [arXiv: 1502.01361] [InSPIRE].

[33] B. Batell et al., Muon anomalous magnetic moment through the leptonic Higgs portal, Phys. Rev. D 95 (2017) 075003 [arXiv:1606.04943] [INSPIRE]. 
[34] G. Cacciapaglia et al., Search for a lighter Higgs boson in Two Higgs Doublet Models, JHEP 12 (2016) 068 [arXiv: 1607.08653] [INSPIRE].

[35] E.J. Chun, S. Dwivedi, T. Mondal and B. Mukhopadhyaya, Reconstructing a light pseudoscalar in the type-X Two Higgs Doublet Model, Phys. Lett. B 774 (2017) 20 [arXiv: 1707.07928] [INSPIRE].

[36] D. Ghosh, R.S. Gupta and G. Perez, Is the Higgs mechanism of fermion mass generation a fact? A Yukawa-less first-two-generation model, Phys. Lett. B 755 (2016) 504 [arXiv: 1508.01501] [INSPIRE].

[37] U. Ellwanger and M. Rodriguez-Vazquez, Discovery prospects of a light scalar in the NMSSM, JHEP 02 (2016) 096 [arXiv:1512.04281] [INSPIRE].

[38] E. Bertuzzo, C. Frugiuele, T. Gregoire and E. Ponton, Dirac gauginos, $R$ symmetry and the 125 GeV Higgs, JHEP 04 (2015) 089 [arXiv: 1402.5432] [INSPIRE].

[39] B. Batell, G.F. Giudice and M. McCullough, Natural heavy supersymmetry, JHEP 12 (2015) 162 [arXiv: 1509.00834] [INSPIRE].

[40] J.L. Evans, T. Gherghetta, N. Nagata and Z. Thomas, Naturalizing supersymmetry with a two-field relaxion mechanism, JHEP 09 (2016) 150 [arXiv: 1602.04812] [INSPIRE].

[41] B. Batell, M.A. Fedderke and L.-T. Wang, Relaxation of the composite Higgs little hierarchy, JHEP 12 (2017) 139 [arXiv:1705. 09666] [INSPIRE].

[42] A. Nelson and C. Prescod-WEinstein, Relaxion: a landscape without anthropics, Phys. Rev. D 96 (2017) 113007 [arXiv:1708.00010] [InSPIRE].

[43] J.R. Espinosa et al., Cosmological Higgs-axion interplay for a naturally small electroweak scale, Phys. Rev. Lett. 115 (2015) 251803 [arXiv:1506. 09217] [INSPIRE].

[44] F. Piazza and M. Pospelov, Sub-eV scalar dark matter through the super-renormalizable Higgs portal, Phys. Rev. D 82 (2010) 043533 [arXiv: 1003.2313] [INSPIRE].

[45] A. Arvanitaki, J. Huang and K. Van Tilburg, Searching for dilaton dark matter with atomic clocks, Phys. Rev. D 91 (2015) 015015 [arXiv: 1405.2925] [INSPIRE].

[46] J.F. Gunion, H.E. Haber, G.L. Kane and S. Dawson, The Higgs hunter's guide, Front. Phys. 80 (2000) 1 [INSPIRE].

[47] M. Bordag, U. Mohideen and V.M. Mostepanenko, New developments in the Casimir effect, Phys. Rept. 353 (2001) 1 [quant-ph/0106045] [INSPIRE].

[48] M. Bordag, G.L. Klimchitskaya, U. Mohideen and V.M. Mostepanenko, Advances in the Casimir effect, Int. Ser. Monogr. Phys. 145 (2009) 1 [INSPIRE].

[49] G.L. Smith et al., Short range tests of the equivalence principle, Phys. Rev. D 61 (2000) 022001 [INSPIRE].

[50] S. Schlamminger et al., Test of the equivalence principle using a rotating torsion balance, Phys. Rev. Lett. 100 (2008) 041101 [arXiv:0712.0607] [INSPIRE].

[51] R. Spero et al., Test of the gravitational inverse-square law at laboratory distances, Phys. Rev. Lett. 44 (1980) 1645 [INSPIRE].

[52] J.K. Hoskins, R.D. Newman, R. Spero and J. Schultz, Experimental tests of the gravitational inverse square law for mass separations from $2 \mathrm{~cm}$ to $105 \mathrm{~cm}$, Phys. Rev. D 32 (1985) 3084 [INSPIRE].

[53] J. Chiaverini et al., New experimental constraints on non-Newtonian forces below 100 microns, Phys. Rev. Lett. 90 (2003) 151101 [hep-ph/0209325] [INSPIRE]. 
[54] C.D. Hoyle et al., Sub-millimeter tests of the gravitational inverse-square law, Phys. Rev. D 70 (2004) 042004 [hep-ph/0405262] [INSPIRE].

[55] S.J. Smullin et al., New constraints on Yukawa-type deviations from Newtonian gravity at 20 microns, Phys. Rev. D 72 (2005) 122001 [Erratum ibid. D 72 (2005) 129901] [hep-ph/0508204] [INSPIRE].

[56] D.J. Kapner et al., Tests of the gravitational inverse-square law below the dark-energy length scale, Phys. Rev. Lett. 98 (2007) 021101 [hep-ph/0611184] [INSPIRE].

[57] J.A. Grifols and E. Masso, Constraints on finite range baryonic and leptonic forces from stellar evolution, Phys. Lett. B 173 (1986) 237 [INSPIRE].

[58] J.A. Grifols, E. Masso and S. Peris, Energy loss from the Sun and red giants: bounds on short range baryonic and leptonic forces, Mod. Phys. Lett. A 4 (1989) 311 [InSPIRE].

[59] J. Redondo and G. Raffelt, Solar constraints on hidden photons re-visited, JCAP 08 (2013) 034 [arXiv: 1305.2920] [INSPIRE].

[60] E. Hardy and R. Lasenby, Stellar cooling bounds on new light particles: plasma mixing effects, JHEP 02 (2017) 033 [arXiv:1611.05852] [INSPIRE].

[61] N. Ishizuka and M. Yoshimura, Axion and dilaton emissivity from nascent neutron stars, Prog. Theor. Phys. 84 (1990) 233.

[62] G. Krnjaic, Probing light thermal dark-matter with a Higgs portal mediator, Phys. Rev. D 94 (2016) 073009 [arXiv: 1512.04119] [INSPIRE].

[63] F. Bezrukov and D. Gorbunov, Light inflaton Hunter's Guide, JHEP 05 (2010) 010 [arXiv:0912.0390] [INSPIRE].

[64] J.D. Clarke, R. Foot and R.R. Volkas, Phenomenology of a very light scalar (100 MeV $<m_{h}<10 \mathrm{GeV}$ ) mixing with the SM Higgs, JHEP 02 (2014) 123 [arXiv:1310.8042] [INSPIRE].

[65] K. Schmidt-Hoberg, F. Staub and M.W. Winkler, Constraints on light mediators: confronting dark matter searches with B physics, Phys. Lett. B 727 (2013) 506 [arXiv: 1310.6752] [INSPIRE].

[66] BNL-E949 collaboration, A.V. Artamonov et al., Study of the decay $K^{+} \rightarrow \pi^{+} \nu \bar{\nu}$ in the momentum region $140<P_{\pi}<199 \mathrm{MeV} / \mathrm{c}$, Phys. Rev. D 79 (2009) 092004 [arXiv:0903.0030] [INSPIRE].

[67] LHCb collaboration, Differential branching fraction and angular analysis of the $B^{+} \rightarrow K^{+} \mu^{+} \mu^{-}$decay, JHEP 02 (2013) 105 [arXiv: 1209.4284] [INSPIRE].

[68] LHCb collaboration, Search for hidden-sector bosons in $B^{0} \rightarrow K^{* 0} \mu^{+} \mu^{-}$decays, Phys. Rev. Lett. 115 (2015) 161802 [arXiv: 1508.04094] [INSPIRE].

[69] L3 collaboration, M. Acciarri et al., Search for neutral Higgs boson production through the process $e^{+} e^{-} \rightarrow Z^{*} H^{0}$, Phys. Lett. B 385 (1996) 454 [INSPIRE].

[70] Delphi, OPAL, Aleph, LeP Working Group for Higgs Boson Searches, L3 collaboration, S. Schael et al., Search for neutral MSSM Higgs bosons at LEP, Eur. Phys. J. C 47 (2006) 547 [hep-ex/0602042] [INSPIRE].

[71] P. Bechtle et al., Probing the standard model with Higgs signal rates from the Tevatron, the LHC and a future ILC, JHEP 11 (2014) 039 [arXiv:1403.1582] [INSPIRE].

[72] KTEV collaboration, A. Alavi-Harati et al., Search for the Decay $K_{L} \rightarrow \pi^{0} \mu^{+} \mu^{-}$, Phys. Rev. Lett. 84 (2000) 5279 [hep-ex/0001006] [INSPIRE]. 
[73] KTeV collaboration, A. Alavi-Harati et al., Search for the rare decay $K_{L} \rightarrow p i^{0} e^{+} e^{-}$, Phys. Rev. Lett. 93 (2004) 021805 [hep-ex/0309072] [INSPIRE].

[74] S. Alekhin et al., A facility to search for hidden particles at the CERN SPS: the SHiP physics case, Rept. Prog. Phys. 79 (2016) 124201 [arXiv:1504.04855] [InSPIRE].

[75] A. Fradette and M. Pospelov, BBN for the LHC: constraints on lifetimes of the Higgs portal scalars, Phys. Rev. D 96 (2017) 075033 [arXiv:1706.01920] [InSPIRE].

[76] K. Choi, H. Kim and T. Sekiguchi, Dynamics of the cosmological relaxation after reheating, Phys. Rev. D 95 (2017) 075008 [arXiv:1611.08569] [INSPIRE].

[77] D. Cadamuro and J. Redondo, Cosmological bounds on pseudo Nambu-Goldstone bosons, JCAP 02 (2012) 032 [arXiv:1110.2895] [InSPIRE].

[78] Yu.N. Pokotilovski, Constraints on new interactions from neutron scattering experiments, Phys. Atom. Nucl. 69 (2006) 924 [hep-ph/0601157] [INSPIRE].

[79] V.V. Nesvizhevsky, G. Pignol and K.V. Protasov, Neutron scattering and extra short range interactions, Phys. Rev. D 77 (2008) 034020 [arXiv:0711.2298] [INSPIRE].

[80] C. Frugiuele, E. Fuchs, G. Perez and M. Schlaffer, Constraining new physics models with isotope shift spectroscopy, Phys. Rev. D 96 (2017) 015011 [arXiv:1602.04822] [INSPIRE].

[81] J.C. Berengut et al., Probing new long-range interactions by isotope shift spectroscopy, Phys. Rev. Lett. 120 (2018) 091801 [arXiv:1704.05068] [InSPIRE].

[82] C. Delaunay, C. Frugiuele, E. Fuchs and Y. Soreq, Probing new spin-independent interactions through precision spectroscopy in atoms with few electrons, Phys. Rev. D 96 (2017) 115002 [arXiv: 1709.02817] [inSPIRE].

[83] A. Hees et al., Violation of the equivalence principle from light scalar dark matter, Phys. Rev. D 98 (2018) 064051 [arXiv: 1807.04512] [INSPIRE].

[84] A. Arvanitaki, S. Dimopoulos and K. Van Tilburg, Resonant absorption of bosonic dark matter in molecules, Phys. Rev. X 8 (2018) 041001 [arXiv:1709. 05354] [INSPIRE].

[85] C. Delaunay, R. Ozeri, G. Perez and Y. Soreq, Probing atomic Higgs-like forces at the precision frontier, Phys. Rev. D 96 (2017) 093001 [arXiv: 1601.05087] [INSPIRE].

[86] B. Döbrich et al., ALPtraum: ALP production in proton beam dump experiments, JHEP 02 (2016) 018 [arXiv: 1512.03069] [InSPIRE].

[87] J.A. Evans, Detecting hidden particles with MATHUSLA, Phys. Rev. D 97 (2018) 055046 [arXiv: 1708.08503] [INSPIRE].

[88] D. Curtin et al., Long-lived particles at the energy frontier: the MATHUSLA physics case, arXiv:1806.07396 [INSPIRE].

[89] J. Liu, Z. Liu and L.-T. Wang, Long-lived particles at the LHC: catching them in time, arXiv: 1805.05957 [INSPIRE].

[90] S. Knapen, T. Lin, H.K. Lou and T. Melia, Searching for axionlike particles with ultraperipheral heavy-ion collisions, Phys. Rev. Lett. 118 (2017) 171801 [arXiv:1607.06083] [INSPIRE].

[91] M. Bauer, M. Neubert and A. Thamm, Collider probes of axion-like particles, JHEP 12 (2017) 044 [arXiv: 1708.00443] [InSPIRE].

[92] I. Brivio et al., ALPs effective field theory and collider signatures, Eur. Phys. J. C 77 (2017) 572 [arXiv: 1701.05379] [INSPIRE].

[93] ALEPH collaboration, R. Barate et al., Search for evidence of compositeness at LEP I, Eur. Phys. J. C 4 (1998) 571 [INSPIRE]. 
[94] SLD Electroweak Group, Delphi, Aleph, SLD, Sld Heavy Flavour Group, OPAL, LEP Electroweak Working Group, L3 collaboration, S. Schael et al., Precision electroweak measurements on the $Z$ resonance, Phys. Rept. 427 (2006) 257 [hep-ex/0509008] [INSPIRE].

[95] ACME collaboration, J. Baron et al., Order of magnitude smaller limit on the electric dipole moment of the electron, Science 343 (2014) 269 [arXiv:1310.7534] [INSPIRE].

[96] J.L. Hewett et al., Fundamental physics at the intensity frontier, arXiv:1205.2671.

[97] LHC Higgs Cross Section Working Group collaboration, J.R. Andersen et al., Handbook of LHC Higgs Cross Sections: 3. Higgs properties, arXiv:1307.1347 [INSPIRE].

[98] CMS collaboration, Precise determination of the mass of the Higgs boson and tests of compatibility of its couplings with the standard model predictions using proton collisions at 7 and $8 \mathrm{TeV}$, Eur. Phys. J. C 75 (2015) 212 [arXiv:1412.8662] [INSPIRE].

[99] CMS collaboration, Combined measurements of the Higgs boson's couplings at $\sqrt{s}=13$ TeV, CMS-PAS-HIG-17-031 (2018).

[100] ATLAS collaboration, Combined measurements of Higgs boson production and decay using up to $80 \mathrm{fb}^{-1}$ of proton-proton collision data at $\sqrt{\mathrm{s}}=13 \mathrm{TeV}$ collected with the ATLAS experiment, ATLAS-CONF-2018-031 (2018).

[101] G. Bélanger et al., Global fit to Higgs signal strengths and couplings and implications for extended Higgs sectors, Phys. Rev. D 88 (2013) 075008 [arXiv: 1306.2941] [INSPIRE].

[102] H. Abramowicz et al., Higgs physics at the CLIC electron-positron linear collider, Eur. Phys. J. C 77 (2017) 475 [arXiv:1608.07538] [INSPIRE].

[103] CLICDP, CLIC collaboration, M.J. Boland et al., Updated baseline for a staged compact linear collider, arXiv: 1608.07537 [INSPIRE].

[104] Z. Chen et al., Cross section and Higgs mass measurement with Higgsstrahlung at the CEPC, Chin. Phys. C 41 (2017) 023003 [arXiv:1601.05352] [InSPIRE].

[105] K. Fujii et al., Physics case for the $250 \mathrm{GeV}$ stage of the International Linear Collider, arXiv: 1710.07621 [INSPIRE].

[106] S. Dawson et al., Working group report: Higgs boson, in the proceedings of the 2013 Community Summer Study on the Future of U.S. Particle Physics: Snowmass on the Mississippi (CSS2013), July 29-August 6, Minneapolis, U.S.A. (2013), arXiv:1310.8361 [INSPIRE].

[107] TLeP Design Study Working Group collaboration, M. Bicer et al., First Look at the Physics Case of TLEP, JHEP 01 (2014) 164 [arXiv:1308.6176] [INSPIRE].

[108] CLICDP collaboration, G. Milutinovic-Dumbelovic, https://indico.in2p3.fr/event/13480/attachments/12226/15031/Gordana_Paris.pdf, (2016).

[109] S. Di Vita et al., A global view on the Higgs self-coupling at lepton colliders, JHEP 02 (2018) 178 [arXiv: 1711.03978] [InSPIRE].

[110] Particle Data Group collaboration, C. Patrignani et al., Review of particle physics, Chin. Phys. C 40 (2016) 100001 [InSPIRE].

[111] A. Freitas, Higher-order electroweak corrections to the partial widths and branching ratios of the $Z$ boson, JHEP 04 (2014) 070 [arXiv:1401.2447] [INSPIRE].

[112] A. Freitas, Numerical multi-loop integrals and applications, Prog. Part. Nucl. Phys. 90 (2016) 201 [arXiv: 1604.00406] [INSPIRE]. 
[113] LHC Higgs CROSS SECTION WORKING GROUP, https://twiki.cern.ch/twiki/pub/ LHCPhysics/CERNYellowReportPageAt1314TeV2014/Higgs_XS_parton_luminosity.pdf.

[114] CMS collaboration, A search for beyond standard model light bosons decaying into muon pairs, CMS-PAS-HIG-16-035 (2015).

[115] ATLAS collaboration, Search for Higgs boson decays to beyond-the-Standard-Model light bosons in four-lepton events with the ATLAS detector at $\sqrt{s}=13 \mathrm{TeV}$, JHEP 06 (2018) 166 [arXiv: 1802.03388] [INSPIRE].

[116] Z. Liu, L.-T. Wang and H. Zhang, Exotic decays of the $125 \mathrm{GeV}$ Higgs boson at future $e^{+} e^{-}$ lepton colliders, Chin. Phys. C 41 (2017) 063102 [arXiv: 1612.09284] [INSPIRE].

[117] CMS collaboration, Search for the exotic decay of the Higgs boson to a pair of light pseudoscalars in the final state with two $b$ quarks and two $\tau$ leptons, CMS-PAS-HIG-17-024 (2017).

[118] CMS collaboration, Search for light bosons in decays of the $125 \mathrm{GeV}$ Higgs boson in proton-proton collisions at $\sqrt{s}=8 \mathrm{TeV}$, JHEP 10 (2017) 076 [arXiv:1701.02032] [INSPIRE].

[119] ATLAS collaboration, Search for Higgs boson decays into a pair of light bosons in the bb $\mu \mu$ final state in pp collision at $\sqrt{s}=13 \mathrm{TeV}$ with the ATLAS detector, arXiv:1807.00539 [INSPIRE].

[120] CMS collaboration, Search for an exotic decay of the Higgs boson to a pair of light pseudoscalars in the final state of two muons and two $\tau$ leptons in proton-proton collisions at $\sqrt{s}=13 \mathrm{TeV}$, arXiv:1805.04865 [INSPIRE].

[121] ATLAS collaboration, Search for the Higgs boson produced in association with a vector boson and decaying into two spin-zero particles in the $H \rightarrow a a \rightarrow 4 b$ channel in pp collisions at $\sqrt{s}=13 \mathrm{TeV}$ with the ATLAS detector, JHEP 10 (2018) 031 [arXiv:1806.07355] [INSPIRE].

[122] ATLAS collaboration, Search for Higgs boson decays into pairs of light (pseudo)scalar particles in the $\gamma \gamma j$ final state in pp collisions at $\sqrt{s}=13 \mathrm{TeV}$ with the ATLAS detector, Phys. Lett. B 782 (2018) 750 [arXiv:1803.11145] [InSPIRE].

[123] R.D. Ball et al., Higgs production in gluon fusion beyond NNLO, Nucl. Phys. B 874 (2013) 746 [arXiv: 1303.3590] [INSPIRE].

[124] M. Bonvini et al., Updated Higgs cross section at approximate $N^{3} L O, J$. Phys. G 41 (2014) 095002 [arXiv: 1404.3204] [INSPIRE].

[125] M. Bonvini, S. Marzani, C. Muselli and L. Rottoli, On the Higgs cross section at $N^{3} L O+N^{3} L L$ and its uncertainty, JHEP 08 (2016) 105 [arXiv: 1603.08000] [INSPIRE].

[126] T. Ahmed et al., Pseudo-scalar Higgs boson production at $N^{3} L O_{A}+N^{3} L L^{\prime}$, Eur. Phys. J. C 76 (2016) 663 [arXiv: 1606.00837] [INSPIRE].

[127] J. Alwall et al., The automated computation of tree-level and next-to-leading order differential cross sections and their matching to parton shower simulations, JHEP $\mathbf{0 7}$ (2014) 079 [arXiv: 1405.0301] [INSPIRE].

[128] CMS collaboration, Search for a low-mass pseudoscalar Higgs boson produced in association with a b $\bar{b}$ pair in pp collisions at $\sqrt{s}=8 \mathrm{TeV}$, Phys. Lett. B 758 (2016) 296 [arXiv: 1511.03610] [INSPIRE].

[129] CMS collaboration, Search for a light pseudoscalar Higgs boson produced in association with bottom quarks in pp collisions at $\sqrt{s}=8 \mathrm{TeV}$, JHEP 11 (2017) 010 [arXiv: 1707.07283] [INSPIRE]. 
[130] M. Casolino et al., Probing a light CP-odd scalar in di-top-associated production at the LHC, Eur. Phys. J. C 75 (2015) 498 [arXiv: 1507.07004] [INSPIRE].

[131] W.-F. Chang, T. Modak and J.N. Ng, Signal for a light singlet scalar at the LHC, Phys. Rev. D 97 (2018) 055020 [arXiv: 1711.05722] [INSPIRE].

[132] R. Mertig, M. Böhm and A. Denner, FEYN CALC: computer algebraic calculation of Feynman amplitudes, Comput. Phys. Commun. 64 (1991) 345 [INSPIRE].

[133] V. Shtabovenko, R. Mertig and F. Orellana, New developments in FeynCalc 9.0, Comput. Phys. Commun. 207 (2016) 432 [arXiv:1601.01167] [InSPIRE].

[134] NNPDF collaboration, R.D. Ball et al., Parton distributions from high-precision collider data, Eur. Phys. J. C 77 (2017) 663 [arXiv:1706.00428] [INSPIRE].

[135] D.B. Clark, E. Godat and F.I. Olness, ManeParse: a Mathematica reader for parton distribution functions, Comput. Phys. Commun. 216 (2017) 126 [arXiv:1605.08012] [INSPIRE].

[136] P. Drechsel, G. Moortgat-Pick and G. Weiglein, Sensitivity of the ILC to light Higgs masses, talk given at the International Workshop on Future Linear Collider (LCWS2017), October 23-27, Strasbourg, France (2018), arXiv:1801.09662 [INSPIRE].

[137] C. Delaunay, G. Perez, H. de Sandes and W. Skiba, Higgs up-down CP asymmetry at the LHC, Phys. Rev. D 89 (2014) 035004 [arXiv:1308.4930] [INSPIRE].

[138] R. Assmann, M. Lamont and S. Myers, A brief history of the LEP collider, Nucl. Phys. Proc. Suppl. 109B (2002) 17 [InSPIRE].

[139] D. Buttazzo, D. Redigolo, F. Sala and A. Tesi, Fusing vectors into scalars at high energy lepton colliders, arXiv:1807.04743 [INSPIRE]. 